\title{
DJ-1 maintains energy and glucose homeostasis by regulating the function of brown adipose tissue
}

\author{
Rong $\mathrm{Wu}^{1,2}$, Xiao-meng Liu ${ }^{3,4}$, Jian-guang Sun ${ }^{1,5}$, Hong Chen ${ }^{1}$, Jun $\mathrm{Ma}^{1,2}$, Meng Dong ${ }^{2,3}$, Shengyi Peng ${ }^{1}$, \\ Ji-qiu Wang ${ }^{6}$, Jian-qing Ding ${ }^{6}$, Dong-hao Li ${ }^{7}$, John R Speakman ${ }^{8,9}$, Guang Ning ${ }^{6}$, Wanzhu Jin ${ }^{3}$, \\ Zengqiang Yuan ${ }^{1}$
}

\begin{abstract}
${ }^{1}$ State Key Laboratory of Brain and Cognitive Sciences, Institute of Biophysics, Chinese Academy of Sciences, Beijing, China; ${ }^{2}$ College of Life Sciences, University of Chinese Academy of Sciences, Beijing, China; ${ }^{3}$ Key Laboratory of Animal Ecology and Conservation Biology, Institute of Zoology, Chinese Academy of Sciences, Beijing, China; ${ }^{4}$ College of life science and agronomy, Zhoukou Normal University, Zhoukou, China; ${ }^{5}$ Sino-Danish Center Neuroscience Program, University of Chinese Academy of Sciences, Beijing, China; ${ }^{6}$ Ruijin Hospital Affiliated to Shanghai Jiaotong University School of Medicine, Shanghai, China; ${ }^{7}$ Key Laboratory of Natural Resources of Changbai Mountain and Functional Molecules, Ministry of Education, Yanbian University, Yanji, China; ${ }^{8}$ State Key Laboratory of Molecular Developmental Biology (Beijing), Institute of Genetics and Developmental Biology, Chinese Academy of Sciences, Beijing, China; ${ }^{9}$ Institute of Biological and Environmental Sciences, University of Aberdeen, Aberdeen, UK
\end{abstract}

DJ-1 protein is involved in multiple physiological processes, including Parkinson's disease. However, the role of DJ-1 in the metabolism is largely unknown. Here we found that DJ-1 maintained energy balance and glucose homeostasis via regulating brown adipose tissue (BAT) activity. DJ-1-deficient mice reduced body mass, increased energy expenditure and improved insulin sensitivity. DJ-1 deletion also resisted high-fat-diet (HFD) induced obesity and insulin resistance. Accordingly, DJ-1 transgene triggered autonomous obesity and glucose intolerance. Further BAT transplantation experiments clarified DJ-1 regulates energy and glucose homeostasis by modulating BAT function. Mechanistically, we found that DJ-1 promoted PTEN proteasomal degradation via an E3 ligase, mind bomb-2 (Mib2), which led to Akt activation and inhibited FoxO1-dependent Ucp1 (Uncoupling protein-1) expression in BAT. Consistently, ablation of Akt1 mitigated the obesity and BAT dysfunction induced by DJ-1 transgene. These findings define a new biological role of DJ-1 protein in regulating BAT function, with an implication of the therapeutic target in the treatment of metabolic disorders. Keywords: BAT; DJ-1; obesity; ubiquitination; Ucp1

Cell Discovery (2017) 3, 16054; doi:10.1038/celldisc.2016.54; published online 14 February 2017

\section{Introduction}

Obesity occurs when energy intake exceeds energy expenditure [1]. Obesity has reached epidemic proportions worldwide and is accompanied by a series of metabolic diseases including type II diabetes, hepatic steatosis and cardiovascular diseases [2]. Increasing energy expenditure, especially promoting brown

Correspondence: Wanzhu Jin

Tel/Fax: +86 10 64806302;

E-mail: jinw@ioz.ac.cn.

or Zengqiang Yuan

Tel/Fax: +86 1066931579 ;

E-mail: zqyuan@ibp.ac.cn

Received 24 November 2016; accepted 28 December 2016 adipose tissue (BAT) thermogenesis [1], has received considerably more attention than inhibiting energy intake and/or absorption as an effective anti-obesity treatment. It has been shown that BAT activity is inversely correlated with body mass index (BMI) and the percentage of body fat [3,4]. Recently, we and other groups have shown that BAT transplantation can improve whole body energy metabolism and glucose homeostasis [5-7].

DJ-1 has been implicated in reproduction [8-10], androgen receptor signaling [11-13] and tumorigenesis $[14,15]$. Recent evidence indicates that mutations in the DJ-1 gene are associated with early-onset Parkinsonism [16]. DJ-1 could also be connected with two other Parkinson disease-related genes; 
Parkin and Pten-induced putative kinase 1 (PINK1) to form the E3 ligase complex, thereby promoting the degradation of unfolded proteins and Parkinsonism [17]. Interestingly, Parkin and PINK1 are reported to be involved in the regulation of lipid metabolism $[18,19]$.

DJ-1-mediated PI3K/Akt activation has been reported to occur in neuronal protection and tumorigenesis [15, 20, 21]. PTEN (phosphatase and tensin homologue deleted on chromosome 10), a putative phosphatase, has a dominant inhibitory role in PI3K/Akt signaling [22]. PTEN transgenic (Tg) mice have reduced body weight, elevated energy expenditure and insulin sensitivity [23, 24]. The activity of BAT is activated in PTEN Tg mice, but impaired in Myf5cre-mediated PTEN knockout (KO) mice [25]. These findings indicate that the PTEN-Akt cascade participates in the regulation of insulin sensitivity and BAT function. However, whether and how DJ-1 is involved in energy and glucose homeostasis and BAT function are largely unknown.

\section{Results}

DJ-1 KO mice have reduced adiposity, increased energy expenditure and insulin sensitivity

No developmental abnormalities were observed in DJ-1 KO mice compared with wild-type (WT) mice. However, we found a significant reduction in body mass in DJ-1 KO mice compared with their WT counterparts from the maturity-onset stage (age 16 weeks) onwards (Figure 1a). Magnetic resonance imaging (MRI) showed an increase in the percentage of lean mass and a reduction in the percentage of body fat in DJ-1 KO mice (Figure 1b). Further examination showed that the reduction in the percentage of body fat in DJ-1 KO mice was mainly due to a reduction in the mass of epididymal white adipose tissue (eWAT), subcutaneous white adipose tissue (sWAT) and brown adipose tissue (BAT), but not other tissues, without lower free fatty acid (FFA) in plasma (Supplementary Figure S1A-D). Consistent with MRI results, histological analysis revealed that lipid droplets in adipose tissues from DJ-1 KO mice were smaller compared with those in WT mice (Figure 1c). These results indicate that deletion of DJ-1 specifically affects adipose tissue composition.

Changes in adiposity are often accompanied with alterations in energy balance. We next examined whether there was any change in energy metabolism in DJ-1 KO mice. Interestingly, we found a significant increase in energy expenditure in DJ-1 KO mice compared with WT mice (Figure 1d), even though there was no significant difference in respiratory quotients (Supplementary Figure S1E), food intake (Supplementary Figure S1F) and physical activity (Supplementary Figure S1G) between two groups. These results suggest that reduced adiposity in DJ-1 KO mice might be due to an increase in energy expenditure.

Alterations in adiposity often affect insulin sensitivity. To clarify this point, we performed glucose tolerance tests (GTT) in WT and DJ-1 KO mice. Consistent with body mass results, there was no change in glucose tolerance in 3-month-old mice between the two groups (Figure 1e and Supplementary Figure $\mathrm{S} 1 \mathrm{H})$. However, glucose tolerance was significantly improved in aged DJ-1 KO mice compared with WT mice (Figure 1e and Supplementary Figure S1I-K). The insulin tolerance tests (ITT) showed the similar results (Figure if and Supplementary Figure S1L-O). These data suggest that progressive improvement of insulin sensitivity in DJ-1 KO mice was due to a reduction in adiposity.

The difference in adiposity between DJ-1 KO and WT mice was further enhanced in the HFD (high-fat diet) model (Figure 1g). After 5-week of HFD, body mass gain was significantly reduced in DJ-1 KO mice $(P<0.05)$ and this difference was much more evident in the long-term HFD treatment $(P<0.001$; Figure 1g). MRI analysis showed that lean mass and body fat mass decreased in DJ-1 KO mice (Supplementary Figure S2A). In particular, the mass of eWAT, sWAT and BAT were markedly reduced in DJ-1 KO mice (Supplementary Figure S2B). Furthermore, histological analysis showed a marked decrease in HFD-induced lipid accumulation in both adipose tissue and the liver in DJ-1 KO mice (Supplementary Figure S2C). Consistent with results from chow diet experiments, energy expenditure was significantly increased in DJ-1 KO mice (Figure $1 \mathrm{~h}$ and Supplementary Figure S2D), and led to improved insulin sensitivity in DJ-1 KO mice during HFD (Figure 1i). Taken together, these results indicate that the increased energy expenditure significantly reduced adiposity and improved insulin sensitivity in DJ-1 KO mice.

\section{Overexpression of DJ-1 triggers obesity and glucose intolerance}

Significant reduction in adiposity and improvement of energy metabolism in DJ-1 KO mice prompted us to determine whether overexpression of DJ-1 is sufficient to initiate obesity. DJ-1 Tg (Transgenic) mice were generated by transgenically overexpressing 

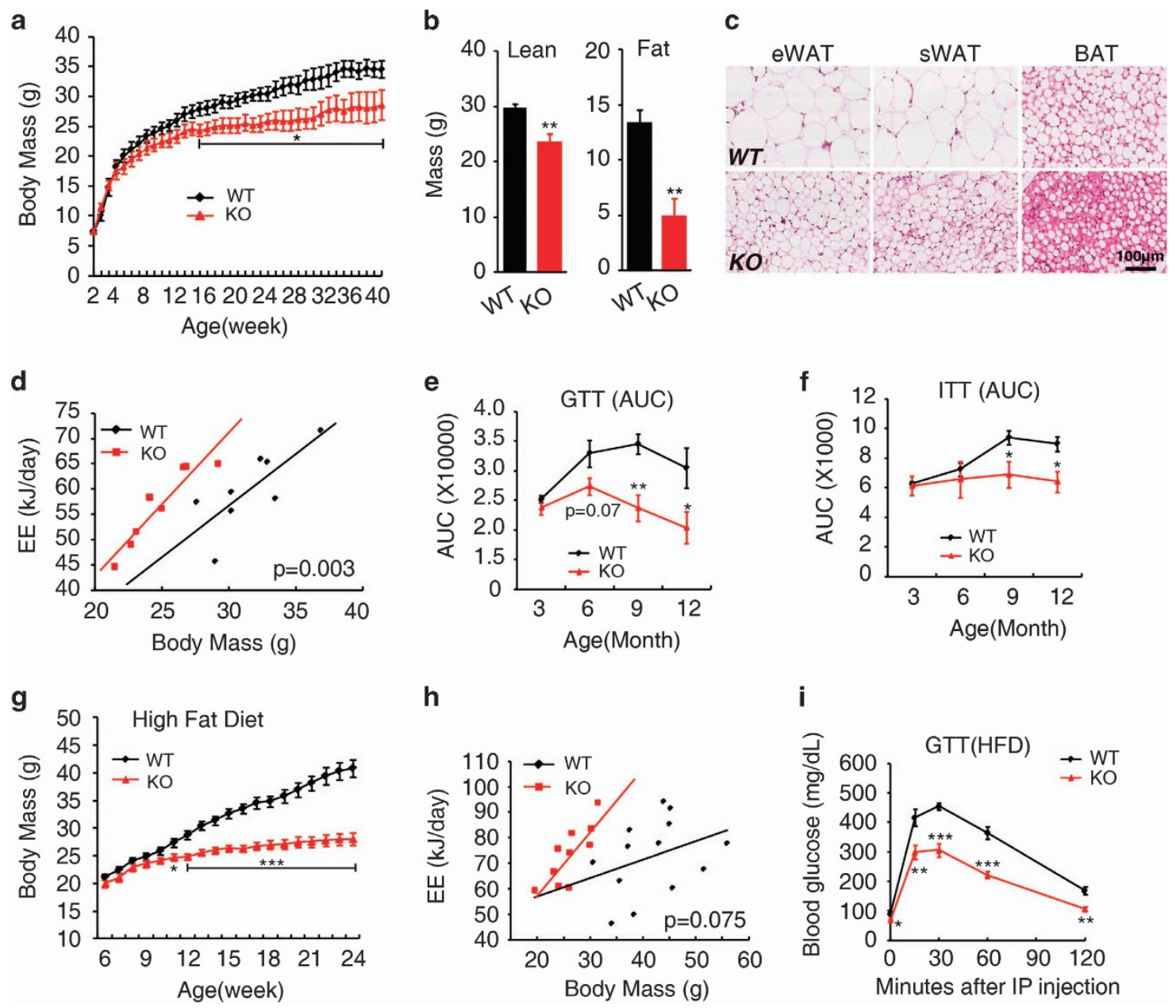

Figure 1 Reduced body mass, increased energy expenditure and improved insulin sensitivity in DJ-1 knockout mice during aging and high-fat diet. (a) Body mass of wild-type (WT) and DJ-1 knockout male mice (KO) fed on a chow diet for 40 weeks (WT, $n=10 ; \mathrm{KO}, n=10$ ). (b) Body lean and fat mass of 12-month-old mice measured by MRI (WT, $n=7 ; \mathrm{KO}, n=6)$. (c) Histology of adipose tissue from 12-month-old mice. eWAT, epididymal white adipose tissue; sWAT, subcutaneous white adipose tissue; BAT, brown adipose tissue. (d) Statistical analysis energy expenditure (EE) by General Linear Model (GLM) and ANCOVA analysis of the 6-month-old male DJ-1 KO and WT mice ( $n=8$ per genotype). (e, f) Quantification of the area under the curve (AUC) of GTT (e) and ITT (f) indicated age ( $n=6$ per genotype). (g) Body mass of mice that were fed with a high-fat diet (HFD) (WT, $n=14$; KO, $n=13$ ). (h) Statistical analysis by General Linear Model (GLM) and ANCOVA analysis of the HFD induced indicated genotype mice energy expenditure (EE) (WT, $n=13$; KO, $n=10$ ). (i) GTT was performed in HFD-fed mice with intraperitoneal injection (IP) $2 \mathrm{~g} \mathrm{~kg}^{-1}$ glucose after fasting $16 \mathrm{~h}(\mathrm{WT}, n=6$; KO, $n=8$ ).

DJ-1 (Supplementary Figure S3A). Transgenic DJ-1 expression was observed in the various tissues (Supplementary Figure S3B).

DJ-1 Tg mice showed no developmental abnormalities. The body mass of DJ-1 Tg mice increased significantly from 10-week age (Figure 2a). MRI analysis showed that DJ-1 Tg mice had increased fat mass, although as there is no significant change in lean mass (Figure 2b). Consistently, there was a marked increase in adipose tissue mass but not other tissues, with similar concentrations of free fatty acid (Supplementary
Figure S3C and D). In addition, histological analysis revealed that lipid droplets in adipose tissues from DJ-1 Tg mice were larger than those in WT mice (Figure 2c).

There was a significant reduction in energy expenditure and increased respiratory quotients in DJ-1 Tg mice (Figure 2d and Supplementary Figure S3E). The defect in energy expenditure was not due to either food intake (Supplementary Figure S3F) or physical activity (Supplementary Figure $\mathrm{S} 3 \mathrm{G}$ ) in DJ-1 $\mathrm{Tg}$ mice. Additionally, we found that there is marked glucose intolerance (Figure 2e and Supplementary Figure S3H) 
a

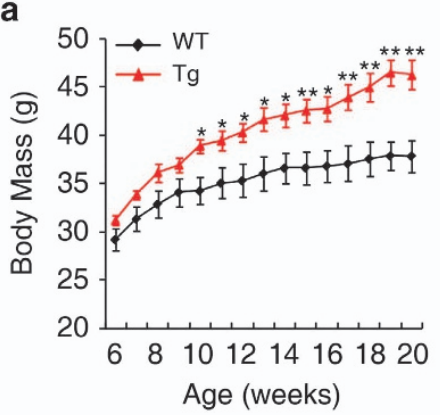

d

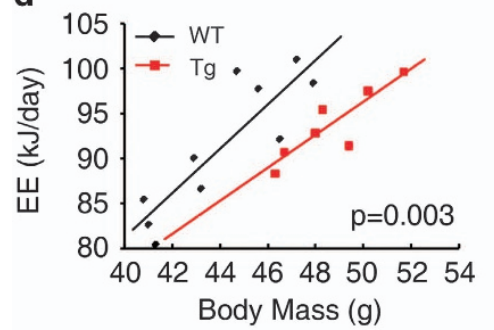

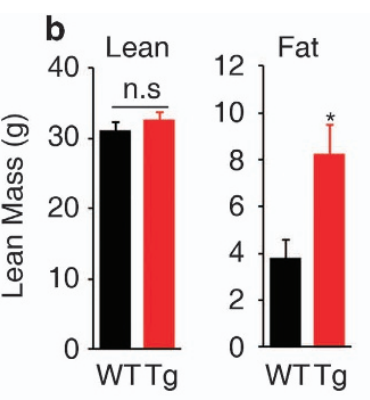

e

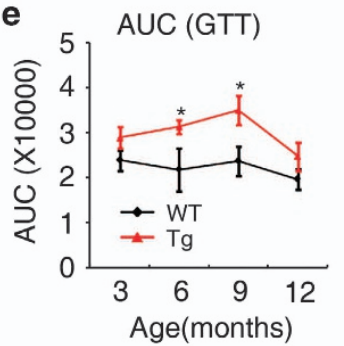

c

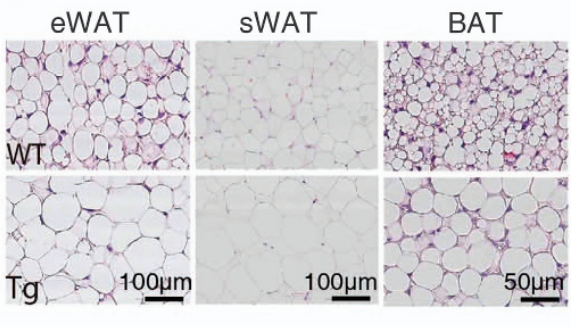

f

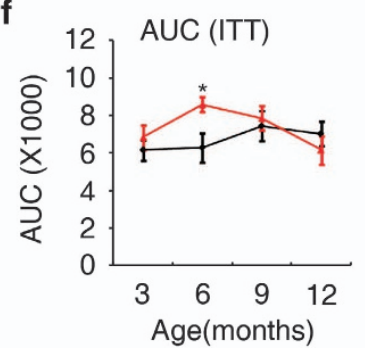

Figure 2 The DJ-1 transgene increases body mass gain and glucose intolerance. (a) Body mass cure of wild-type (WT) and DJ-1 transgenic mice (Tg, Line 13 CD-1 background) fed on a chow diet (WT, $n=9 ; \mathrm{Tg}, n=10$ ). (b) Body lean and fat mass measured by MRI (WT, $n=6$; Tg, $n=6$ ). (c) HE staining of eWAT, sWAT and BAT from 6-month-old mice. (d) Statistical analysis by General Linear Model (GLM) and ANCOVA analysis of the DJ-1 Tg and WT mice energy expenditure (EE) (WT, $n=10 ; \mathrm{Tg}, n=7$ ). (e) and (f) Quantification on the area under the curve (AUC) of GTT (e) and ITT (f) indicated age $(n=6-12)$.

in DJ-1 Tg mice. Insulin tolerance tests showed DJ-1 transgenic had a little effect on insulin sensitivity (Figure 2f and Supplementary Figure S3I). Taken together these results show that expression of the DJ-1 transgene is sufficient to induce obesity and glucose intolerance.

\section{DJ-1 regulates Ucp1 expression in brown adipocytes}

Our finding that DJ-1 KO increases energy expenditure and improves insulin sensitivity, whereas DJ-1 Tg confers the opposite phenotype, strongly suggests that DJ-1 may be involved in energy metabolism. BAT is known to consume fatty acids and glucose to contribute energy metabolism [26]. Interestingly, we found that Ucp1, specifically expressed in BAT, was increased markedly in DJ-1 KO mice, although the expression of other fatty acid oxidation-related genes was not altered (Figure 3a). In contrast, BAT-specific genes, including Ucp1, Prdm16 and PGC1 $\alpha$, were markedly decreased in DJ-1 Tg mice (Figure 3b). Immunohistochemical analysis further confirmed that Ucp1 protein levels increased in DJ-1 KO mice and decreased in DJ-1 transgenic mice (Figure 3c). These results indicate that DJ-1 may be involved in the regulation of Ucp1 expression.
Sympathetic outflow from ventral hypothalamus to BAT and WAT controls the expression of thermogenic genes and heat production in brown and beige fat [27]. To test whether ventral hypothalamus controls the sympathetic drive to brown fat, we measured the expression levels of genes encoding for orexigenic and anorexigenic neuropeptides in the ventral hypothalamus. Levels of Agrp, Npy, Pomc, leptin receptor (Leprb) and Signal transducer and activator of transcription 3 (Stat3) were not significant different among the genotypes (Supplementary Figure S4A and B). In addition, we examined the mRNA level of tyrosine hydroxylase (TH), a marker of sympathetic nerve, which has no significant change in BAT from DJ-1 KO or DJ-1 Tg mice compared with their wild-type controls (Supplementary Figure S4C). These results indicated thermogenic Ucp1 expression was not regulated by sympathetic outflow in DJ-1 KO or Tg mice. In addition, we explored whether DJ-1 knockout altered the lipolysis-related epinephrine and norepinephrine signaling in WAT and BAT. We found that DJ-1 KO had no significant effect in the phosphorylation of hormone sensitive lipase (HSL), which is a putative target of epinephrine and norepinephrine signaling, in both BAT and WAT (Supplementary Figure S4D). 
To investigate whether DJ-1 is involved in BAT differentiation, primary brown adipose precursors were isolated from newborn WT and DJ-1 KO mice followed by in vitro BAT differentiation assays. There was a significant increase of pre-adipocyte differentiation ability in DJ-1 KO BAT (Supplementary Figure S4E). The expression of BAT marker genes, including Ucp1 and Prdm16, were markedly increased in differentiated BAT cells from DJ-1 KO mice (Supplementary Figure S4F). Accordingly, DJ-1 transgene confers a significant reduction of pre-adipocyte differentiation ability (Supplementary Figure S4E). The expression of BAT marker genes was markedly decreased in differentiated BAT cells from DJ-1 $\mathrm{Tg}$ mice (Supplementary Figure S4G). Taken together, DJ-1 regulates Ucpl expression in cell autonomous manner.

\section{DJ-1 is involved in the maintenance of BAT functional integrity}

Recently, BAT transplantation has been shown to improve energy expenditure and glucose homeostasis $[6,7]$. We next investigated whether DJ-1 is involved in BAT functional integrity through BAT transplantation experiments. WT mice were subcutaneously transplanted with BAT from WT or DJ-1 KO mice and followed with HFD treatment. Compared with transplantation of WT BAT, transplantation of DJ-1 KO BAT significantly ameliorated HFD-induced body mass gain (Figure 3d). Consistent with our recent study [7], fat and liver mass were significantly decreased after WT or DJ-1 KO BAT transplantation (Supplementary Figure S5A). The size of endogenous brown adipocytes was smaller in mice transplanted with DJ-1 KO BAT than in those transplanted with WT BAT or sham operated mice upon HFD treatment (Figure 3e), a phenomenon often observed in active BAT. There was no difference in the size of adipocytes in eWAT and sWAT (Supplementary Figure S5B). In parallel, transplantation of DJ-1 KO BAT markedly reversed HFD-induced hepatic steatosis compared with the sham control, although WT BAT transplantation had an intermediate rescue effect (Supplementary Figure S5B). Consistent with reports that exogenous BAT can enhance the function of endogenous BAT $[7,28]$, transplantation of DJ-1 KO BAT significantly induced Ucp1 expression in endogenous BAT, as determined by immunohistochemistry and Western blotting (Figure 3e). Further GTT and ITT analysis showed that there are significant improvements in glucose tolerance and insulin sensitivity after BAT transplantation (Figure $3 \mathrm{f}$ and Supplementary Figure S5C).
In contrast to transplantation of DJ-1 KO BAT, we found DJ-1 Tg BAT transplantation failed to improve the functional integrity of endogenous BAT (Figure $3 \mathrm{~g}$ and $\mathrm{h}$ ). DJ-1 $\mathrm{Tg}$ mice were used as recipients, and subcutaneously transplanted with BAT from WT or DJ-1 Tg mice. WT BAT transplantation significantly reduced obesity compared with DJ-1 Tg BAT (Figure 3g, Supplementary Figure S5D and E). Immunohistochemistry and western blotting showed that transplanting WT BAT significantly increased endogenous BAT Ucp1 expression. However, transplanting DJ-1 Tg BAT significantly reduced Ucp1 expression (Figure $3 \mathrm{~h}$ ). In addition, we observed that WT BAT transplantation markedly improved glucose homeostasis compared with transplantation of DJ-1 Tg BAT (Figure 3i and Supplementary Figure S5F). Taken together, these results indicate that DJ-1 is important for BAT functional integrity.

\section{DJ-1 regulates Ucpl expression through PTEN-Akt- FoxO1 signaling cascade}

We next explored the molecular mechanism underlying DJ-1 regulation of BAT functional integrity. A previous report demonstrated that the PTEN-AktFoxO1 pathway regulates Ucp1 expression in BAT [23]. DJ-1 has also been shown to negatively regulate PTEN protein levels in cancer cells [15]. Hence, we hypothesized that the DJ-1-PTEN-Akt-FoxO1 signaling cascade might regulate Ucp1 expression in BAT. We found that PTEN protein levels were significantly increased in BAT from DJ-1 KO mice (Figure 4a), whereas decreased in BAT from DJ-1 Tg mice (Figure 4a). Accordingly, Akt-mediated phosphorylation levels of FoxO1 decreased in DJ-1 KO BAT and increased in DJ-1 Tg BAT (Figure 4a). Further, we found Ucp1 transcription was markedly increased by DJ-1 knockdown and decreased by DJ-1 overexpression (Figure 4b). Chromatin immunoprecipitation (ChIP) in vivo analysis showed that FoxO1 is enriched on the Ucp1 promoter, which is increased in BAT of DJ-1 KO mice but decreased in BAT of DJ-1 Tg mice (Figure 4c). These results indicated that DJ-1 regulates Ucp1 expression via Akt signaling in BAT.

\section{DJ-1 promotes PTEN degradation through Mib2}

It has been showed that DJ-1 negatively regulates PTEN [15], but the molecular mechanism is largely unknown. We next investigated how DJ-1 participated in the regulation of PTEN. There was no significant difference of PTEN mRNA levels between DJ-1 Tg or DJ-1 KO mice and WT mice (Figure 4d). Interestingly, we found that DJ-1 interacts with PTEN in vitro and 
in vivo (Figure $4 \mathrm{e}$ and $\mathrm{f}$ ). Protein half-life analysis showed that overexpression of DJ-1 reduced PTEN stability (Figure 4g). Previous reports have shown that PTEN can be degraded via the proteasomal pathway
$[29,30]$. Here we also found that PTEN ubiquitination is increased by DJ-1 overexpression (Figure 4h), although as reduced by DJ-1 knockdown in brown adipose cells (Figure 4i). a

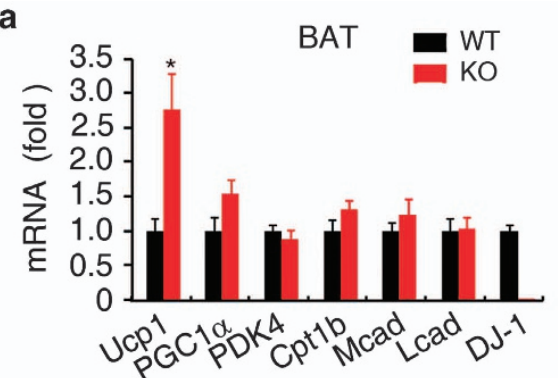

d

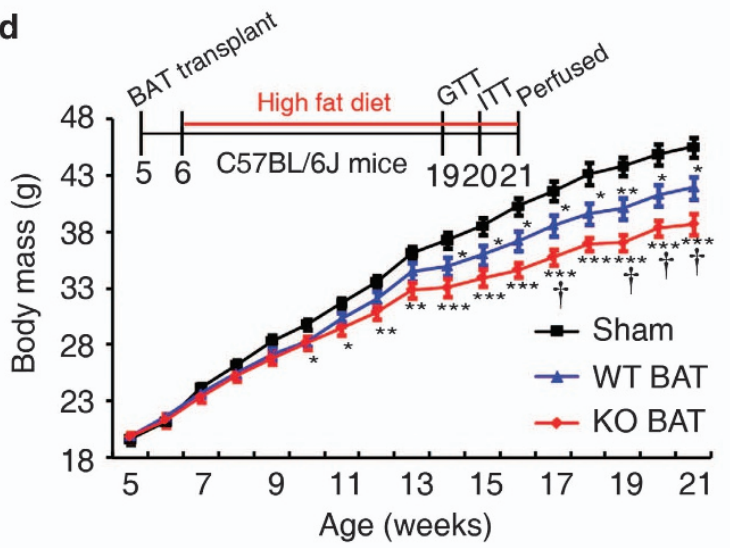

e

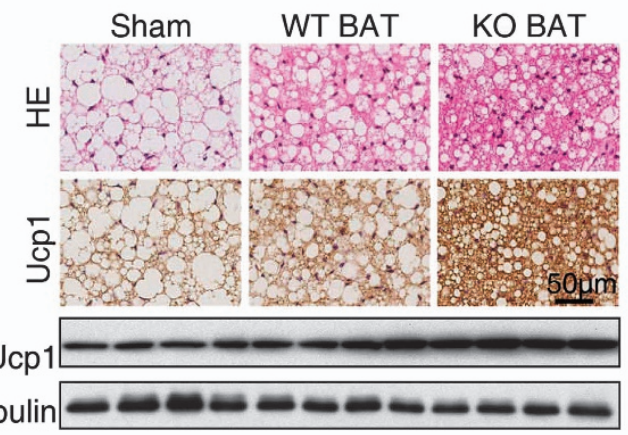

f

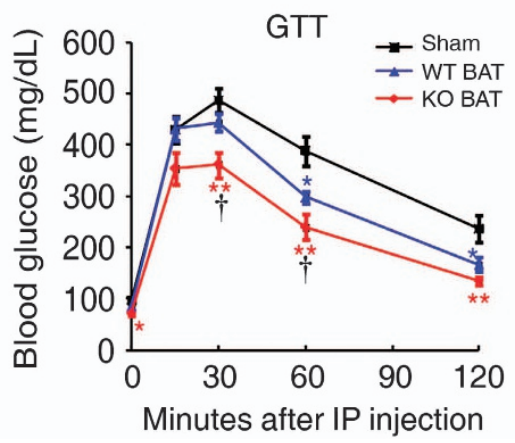

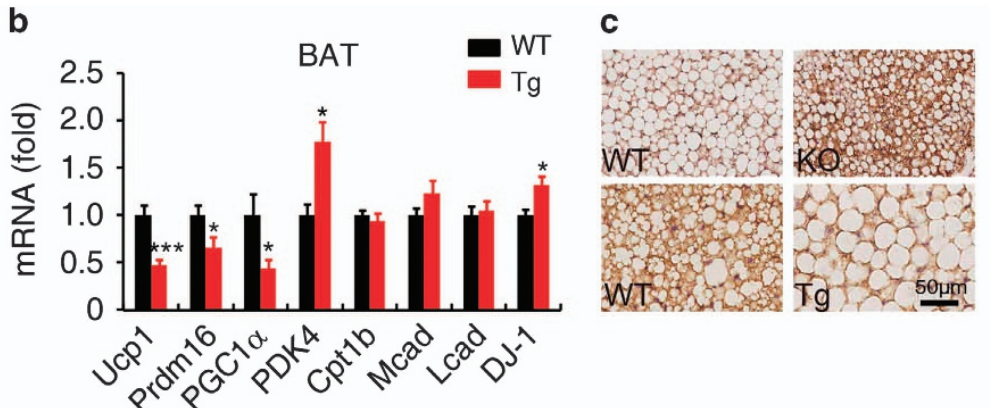

g

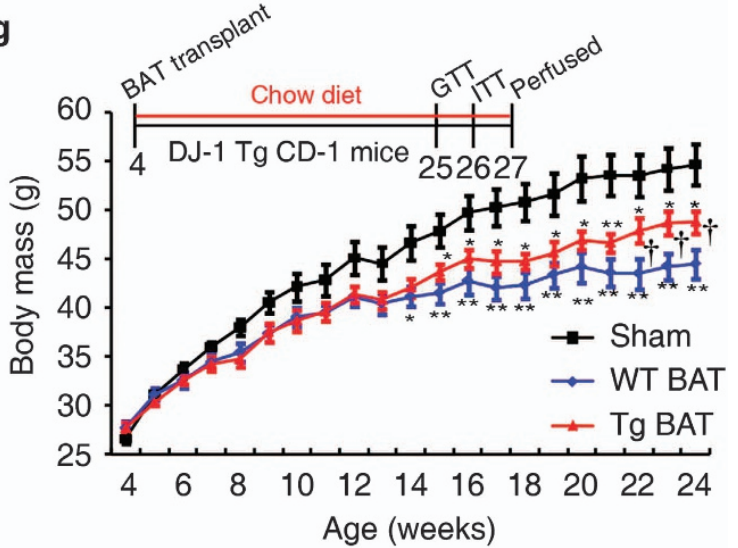

h

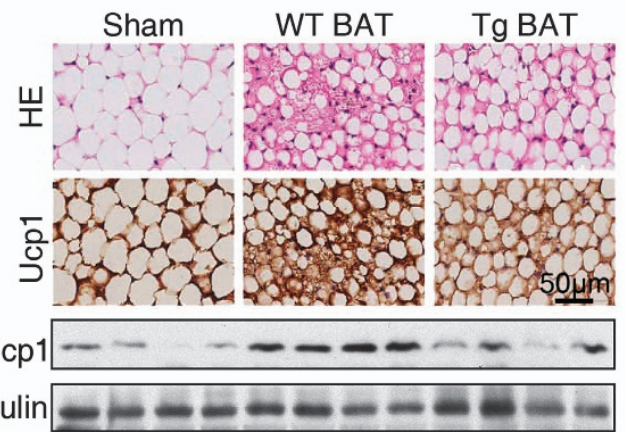

i

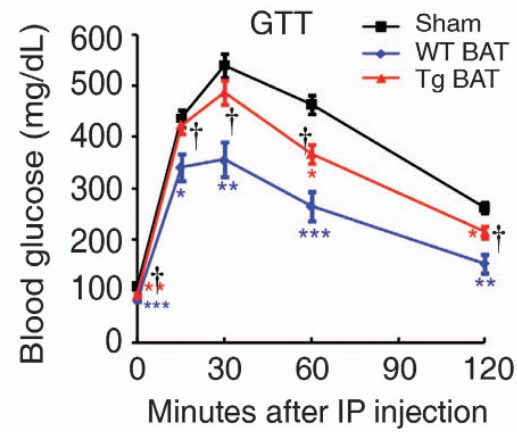


It was demonstrated that DJ-1 formed complex with PINK1/Parkin and promotes unfolded protein degradation [17]. To further explore the mechanism underlying DJ-1-mediated PTEN ubiquitination in BAT, we screened the potential E3 ligases that interact with DJ-1 and identified two E3 ligase, mind bomb-2 (Mib2) and smurf1 (Supplementary Figure S6A). We confirmed the interaction of DJ-1 and Mib2 in BAT and cells (Figure 5a, Supplementary Figure S6B and C) and interestingly, Mib2 also interacted with PTEN (Figure 5b and Supplementary Figure S6D). We also observed both DJ-1 and PTEN interacted with ankyrin repeat domain of Mib2 (Supplementary Figure S6E-G). Interestingly, DJ-1 could significantly promote auto-ubiquitination of Mib2 (Figure 5c). Next we found that Mib2, not Smurf1, was a specific E3 ligase for PTEN ubiquitination (Figure 5d and e). Moreover, PTEN ubiquitination is significantly increased by Mib2 overexpression and reduced by Mib2 knockdown in brown adipocytes (Figure $5 \mathrm{f}$ and $\mathrm{g}$ ). Furthermore, Mib2 with RING domain deletion failed to promote PTEN ubiquitination (Figure 5h), indicating that Mib2-mediated PTEN ubiquitination depends on Mib2 E3 ligase activity. On the other hand, mutation of PTEN ubiquitination site K13 or K289 significantly blocked Mib2-mediated PTEN ubiquitination (Supplementary Figure $\mathrm{S} 6 \mathrm{H}$ ), indicating DJ-1 promoted PTEN ubiquitination through K13 and K289. In addition, DJ-1 could promote Mib2mediated PTEN ubiquitination in vitro (Figure 5i). Importantly, DJ-1 overexpression-induced PTEN ubiquitination could be blocked by Mib2 knockdown (Figure 5j). Finally, we found that PTEN protein level and Ucp1 expression were decreased by Mib2 overexpression, whereas markedly increased by Mib2 knockdown in brown adipocytes (Figure 5k and 1). Collectively, these results demonstrate that DJ-1 promotes PTEN degradation through Mib2 and
DJ-1/Mib2/ PTEN-Akt-FoxO1 pathway regulates Ucp1 transcription in brown adipocytes.

\section{Ablation of Akt1 mitigates the obesity and BAT dysfunction induced by DJ-1 transgene}

To further confirm whether DJ-1 regulates Ucp1 transcription through PI3K-Akt signaling in vivo, we investigated the effect of PI3K on Ucp1 expression and we found that four different PI3K inhibitors, including LY294002, GDC-0941, PI103, BYL-719 could increase the Ucp1 transcription in brown adipocytes (Supplementary Figure S7A). Moreover, oral administration of BYL-719, a PI3K $\alpha$ inhibitor [31], resulted a significant upregulation of Ucp1 and the fatty acid oxidation-related genes in mice (Figure 6a-c). These results suggest that inhibition of PI3K enhances BAT activity.

There are three isoforms of Akt. Akt3 KO mice has overt developmental defect in brain [32]. Knockout of Akt2 led to insulin resistance and diabetes mellitus $[33,34]$. Akt2 is a dominant isoform in adipocytes, but we found no obvious alteration in body mass and energy expenditure in Akt2 KO mice (Supplementary Figure S7B and C). Accordingly, Akt2 deletion has no effect on Ucp1 expression (Supplementary Figure S7D and E). Consistent with previous study [35, 36], we found that there was a significant reduction in body mass and fat mass in Akt1 $\mathrm{KO}$ mice compared with their WT littermates (Figure 6d and e and Supplementary Figure S7F). We next asked whether Akt1 contributes to DJ-1-mediated obesity and we found that Akt1 deletion significantly decreased DJ-1 transgene-induced obesity (Figure 6d and e and Supplementary Figure S7F).

There is no change of energy expenditure between Akt1 KO and WT mice by General Linear Model (GLM) and ANCOVA analysis, which might be due to the analysis is not so sensitive in the mass of the

Figure 3 DJ-1 maintains BAT function. (a) Gene expression in BAT from 12-month-old WT and DJ-1 KO mice ( $n=6$ per genotype). (b) Gene expression in BAT from 4-week-old WT and DJ-1 transgenic mice (WT, $n=6$; TG, $n=7$ ). (c) Ucp1 immunostaining of BAT from indicated genotype mice, upper panel tissues from 12-month-old mice and bottom panel tissues from 6-month old. (d) Body mass of mice with sham operation, BAT transplantation from wild-type (WT BAT) and DJ-1 KO (KO BAT) mice (sham, $n=11$; WT BAT, $n=13$; KO BAT, $n=8$ ). (e) HE (upper panel), Ucp1 immunostaining (middle panel) and immunoblotting (bottom panel) of endogenous BAT from sham operation, WT BAT transplantation and KO BAT transplantation mice. (f) GTT assays of 19-week-old and HFD-induced recipient mice (sham, WT BAT or DJ-1 KO BAT). (g) Body mass curve of the receiver mice of WT BAT or Tg BAT transplantation or sham $(n=6$ per group). WT BAT or Tg BAT was transplanted subcutaneously with the BAT from wild type or DJ-1 transgenic mice. (h) HE (upper panel), Ucp1 immunostaining (middle panel) and immunoblotting (bottom panel) of the endogenous BAT from the groups indicated. (i) GTT assay results for 25-week-old DJ-1 transgenic mice transplanted with BAT. (Data are shown as the mean \pm s.e.m. NS, non significant, ${ }^{\star} P<0.05$, ${ }^{* *} P<0.01$, ${ }^{* * *} P<0.001$ in comparison with sham, ${ }^{\dagger} P<0.05,{ }^{\dagger \dagger} P<0.01$ in comparison with WT BAT, determined using the two-tailed Student's $t$-test). 
a

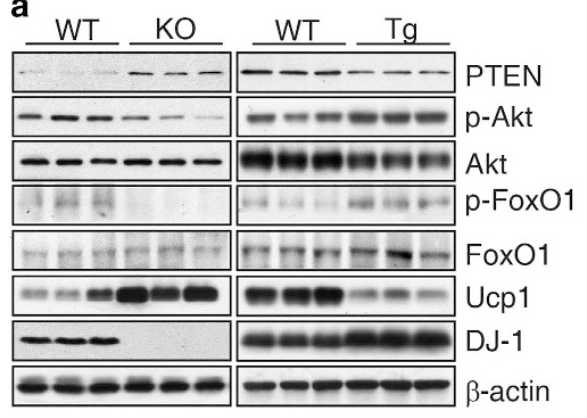

e

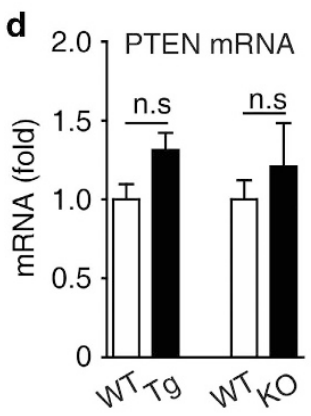

g

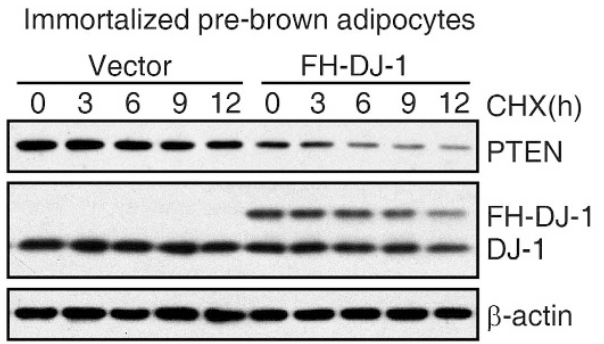

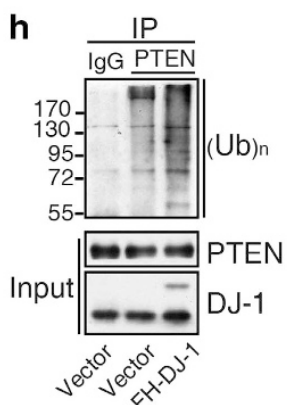
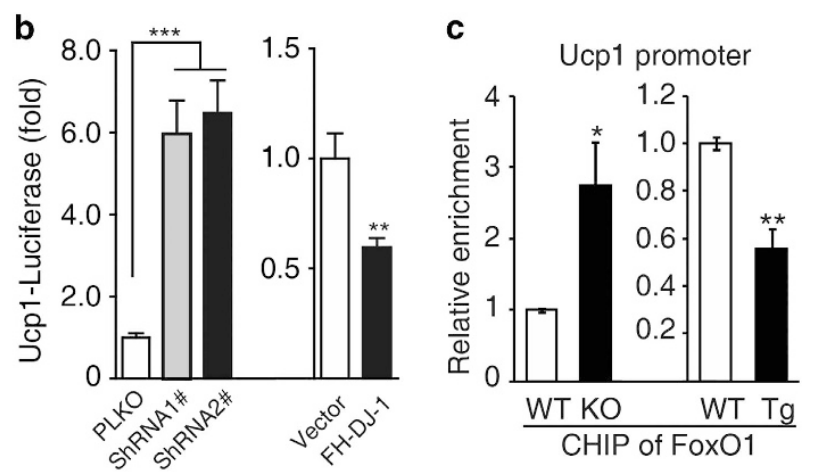

f
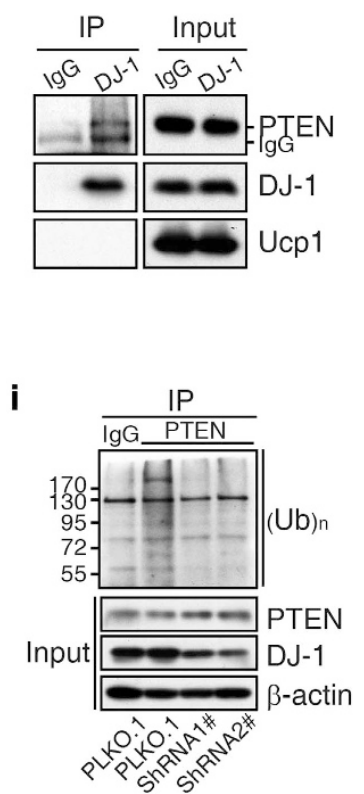

Figure 4 DJ-1 regulates Ucp1 expression by promoting PTEN degradation in brown adipocytes. (a) Lysates of BAT from 12 month-old indicated genotype mice and subjected to immunoblotting with the indicated antibodies. (b) DJ-1 was stably knocked down by lentivirus shRNA or overexpressed with retrovirus in immortalized brown adipose precursors. Ucp1-luciferase reporter activity was examined (Student's $t$-test, ${ }^{* *} P<0.01,{ }^{* * *} P<0.001, n>4$ ). (c) ChIP analysis of FoxO1 on the Ucp1 promoter in BAT from indicated genotype mice $\left(n=3\right.$ per group, Student's $t$-test, ${ }^{*} P<0.05$, $\left.{ }^{* *} P<0.01\right)$. (d) PTEN mRNA levels in BAT from 4week-old DJ-1 transgenic mice and 12-month-old DJ-1 KO mice and their controls ( $n=6-7$ per group). (e) GST-DJ-1 immobilized on glutathione-Sepharose beads pulls down recombinant His tagged PTEN. Pull downs were immunoblotted with anti-PTEN. (f) Lysates of BAT were immunoprecipitated with DJ-1 antibody followed by immunoblotting with PTEN antibody. (g) Cells stably transfected with FH (Flag and HA) tagged DJ-1 or the control vector were treated with $100 \mathrm{\mu g} \mathrm{ml}^{-1} \mathrm{CHX}$ for the number of hours indicated. Equal amounts of total protein lysates were immunoblotted with PTEN or $\beta$-actin antibody. (h, i) Endogenous PTEN ubiqutination was examined in brown adipocytes with overexpression FH tagged DJ-1 (h) or lentivirus vector PLKO.1 vectormediated DJ-1 knockdown (i) after MG132 treatment $8 \mathrm{~h}$.

WT group don't overlap circumstance (Figure 6f). However, Akt1 KO display increased Ucp1 expression (Figure $6 \mathrm{~g}$ and $\mathrm{h}$ ). Moreover, Akt1 $\mathrm{KO}$ restored the energy expenditure of DJ-1 Tg mice (Figure 6f), although no effects on food intake (Supplementary Figure S7G). Furthermore, we found that Akt1 KO reversed DJ-1 transgene-induced reduction of Ucp1 levels (Figure 6g and h). Accordingly, Akt1 deletion improved glucose tolerance (Figure 6i) but not insulin tolerance tests (Supplementary Figure S7H). To further clarify tissue-specific function of Akt1 in BAT, we generated BAT-specific KO mice by crossing conditional Akt1 $\mathrm{KO}(\mathrm{cKO})$ with $M y f 5$-cre mice $[25,37]$. We observed that Aktl deficiency in BAT significantly reduced body mass and adiposity (Figure $7 \mathrm{a}-\mathrm{c}$ ) and increased Ucp1 expression in BAT (Figure 7c and d). 
In addition, Akt1 BAT-specific deletion improved glucose tolerance (Supplementary Figure S7I) but not insulin tolerance tests (Supplementary Figure S7J).

Taken together, we demonstrated that DJ-1-Mib2PTEN-Akt-FoxO1 signaling pathway regulates Ucp1 transcription and the functional integrity of BAT, which is critical for energy balance and glucose homeostasis (working model in Figure 7e).

\section{Discussion}

In this study, we show that DJ-1 maintains energy and glucose homeostasis by modulating the function of BAT and elucidate its molecular mechanism: (i) results from both gain-of-function (DJ-1 transgene) and lossof-function (DJ-1 knockout) experiments demonstrate that DJ-1 regulates energy and glucose metabolism through modulating BAT function. (ii) BAT transplantation experiments further confirmed the tissuespecific function of DJ-1 in regulating BAT activity. (iii) Mechanistically, we found that DJ-1/Mib2 promote PTEN degradation, which in turn activates Akt and inhibits FoxO1-dependent transcription of Ucp1 in BAT.

DJ-1 was first identified as an oncogene [15]. Subsequent studies explored the multifaceted functions of DJ-1 in reproduction [8-10] and Parkinson's diseases [16] as well as tumorigenesis [15]. Recently, DJ-1 has been linked to metabolism [38-41]. However, there have not been any previous systematic and comprehensive studies on the function and molecular mechanism of DJ-1 in energy and glucose homeostasis. In particular, the role of DJ-1 in BAT function has remained a mystery. BAT, which has a major role in energy consumption and thermogenesis, can be activated through the sympathetic nervous system upon cold exposure or nutritional excess treatment, increasing Ucp1 expression and energy expenditure [42]. In this report, we have systematically investigated the role and mechanism of DJ-1 in metabolism, especially in maintaining BAT function.

We found that DJ-1 deficiency increased energy expenditure and improved insulin sensitivity whereas transgenic expression of DJ-1 induced reduced energy consumption and glucose intolerance by alteration BAT activity. To our knowledge, this is the first study indicating that DJ-1 regulates BAT function and metabolic homeostasis. Contrast with our findings, recently, Jain D et al. [38] reports DJ-1 deletion induced glucose intolerance due to reduced islet beta cell and levels of insulin in 12-13 weeks old or 8 weeks old plus 2 weeks of HFD, In our study, glucose tolerance tests were performed from 3 months to 12 months old age, and we found that DJ-1 KO exhibits age-dependent glucose tolerance, although as DJ-1 $\mathrm{Tg}$ mice developed the age-dependent glucose intolerance due to alterations in BAT activity. It is likely that the improved insulin sensitivity was secondary to increased energy expenditure. Therefore, we proposed that the age is critical factor for maintaining glucose homeostasis in these mice.

During our manuscript was preparation, Shi SY et al. [41] reported that DJ-1 deletion modulates muscle ROS levels, thereby promoting mitochondrial uncoupling by increased Ucp3 expression. However, we found that the levels of Ucp3 expression had no significant changes in skeletal muscle under chow diet condition (data not shown). This difference may be explained by the fact that, in the present study, we analyzed skeletal Ucp3 expression at age of 12 months with chow diet condition, although Shi et al. analyzed at young age maximum at 5 month old with HFD condition. It has been shown that high-fat diet could affect the expression of uncoupling proteins expression [43]. DJ-1 modulates HFD-induced ROS production particularly in the skeletal muscle then Ucp3 can be activated by ROS [41, 44]. Therefore, it is not surprising that Ucp3 expression has no change under chow diet, although is increased in HFD in DJ-1 KO mice. In addition, Shi SY et al. did not observe a significant difference on Ucp1 expression in BAT between genotypes by using female mice fed with 3-month HFD treatment. However, we did observe the significant changes in Ucp1 expression in BAT at 12-month-old male mice at chow diet condition (Figure $3 \mathrm{a}-\mathrm{c}$ and Figure 4a). The difference might be due to the difference in age and/or diet condition. Here, we argue that the expression of Ucp1 in BAT is regulated by DJ-1 in physiological condition, although the expression of Ucp3 in muscle is affected by pathological stimulation, including HFD. Interestingly, both studies reached a similar conclusion that DJ-1 deficiency improves glucose tolerance and DJ-1 maintains glucose homeostasis through regulating the whole body energy metabolism in different tissues under physiological and/or pathophysiological condition.

Extensive studies have shown that BAT functions as a thermogenic tissue by increasing energy expenditure and thus may be a therapeutic avenue for the treatment of obesity [1]. BAT transplantation has recently been shown to improve whole body metabolism, especially the maintenance of glucose homeostasis [5-7]. In our BAT transplantation experiments, we found that transplantation of DJ-1 KO BAT significantly 
a
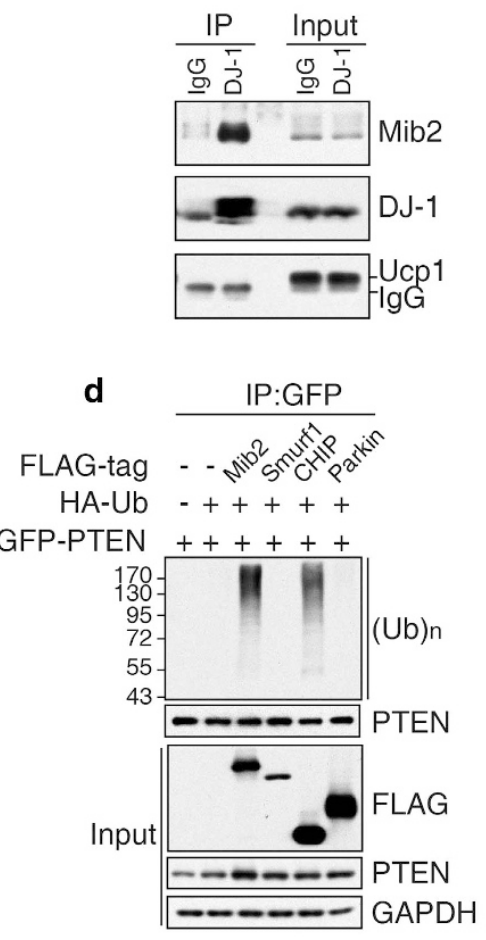

g
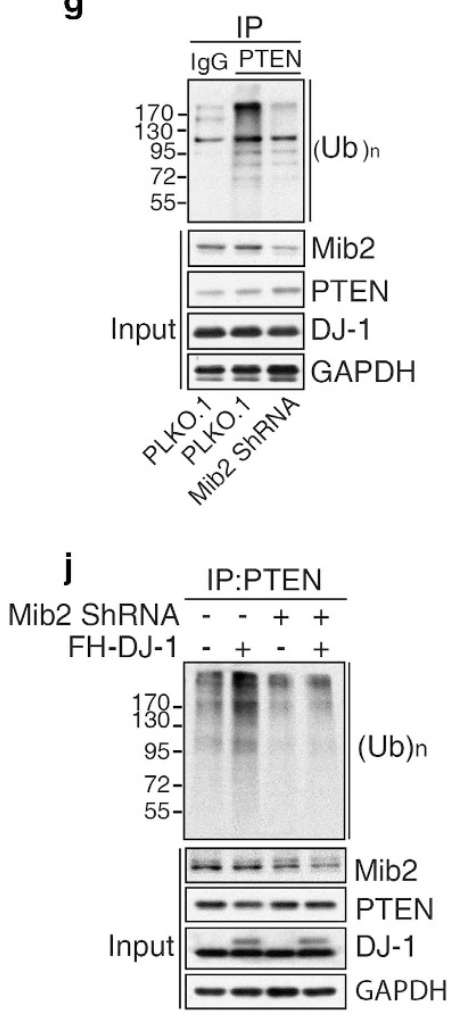

b
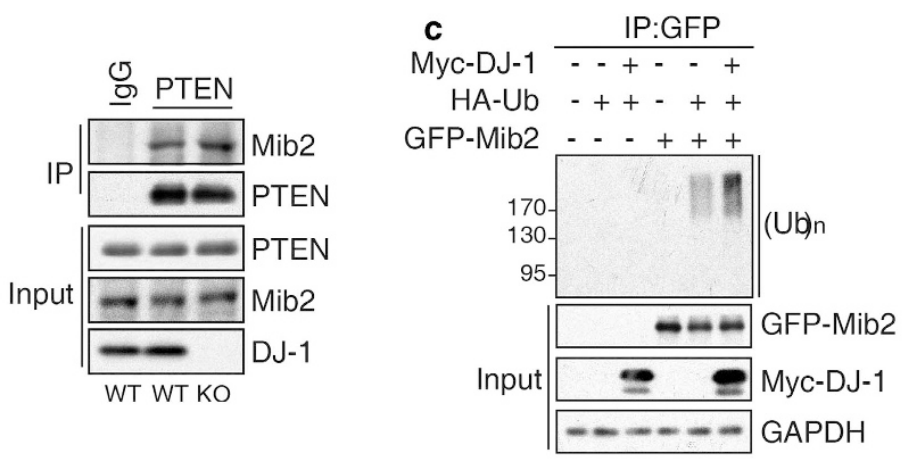

e $\mathrm{E} 1 \quad-\quad++++++$

E2 - - + ++++

Ub - - + ++++

His-PTEN -+++++++

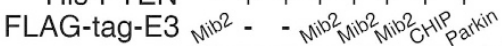
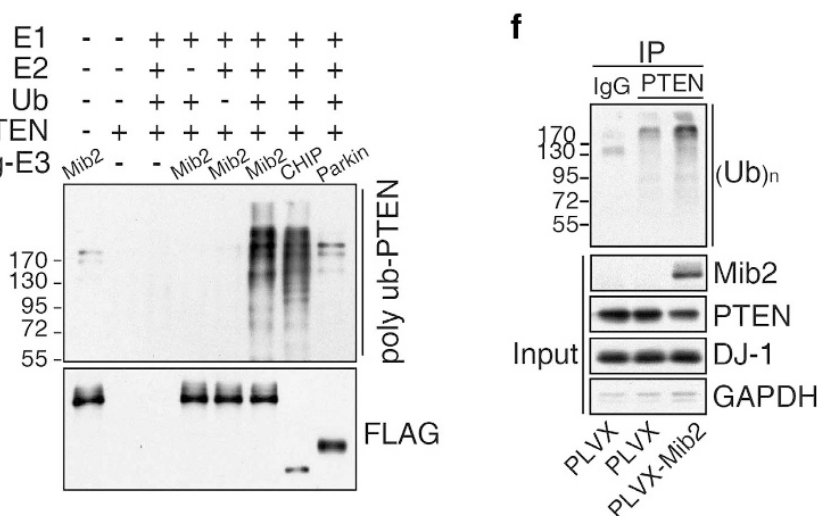

h

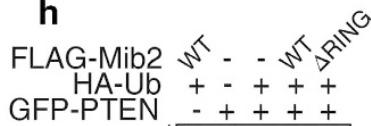

$\begin{aligned} \text { GFP-PTEN }-++++ & +\end{aligned}$

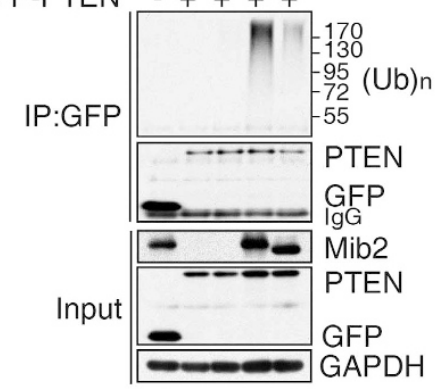

i

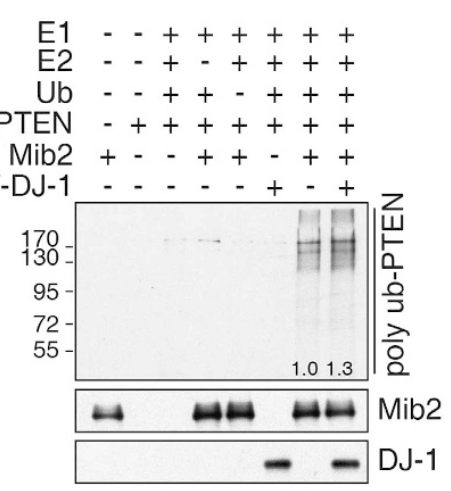

k

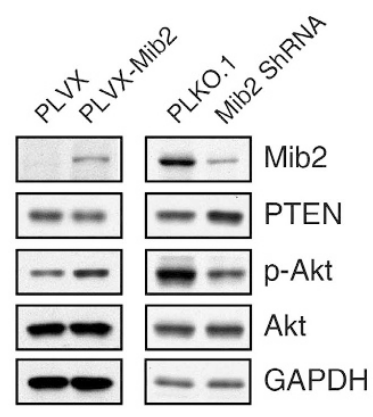

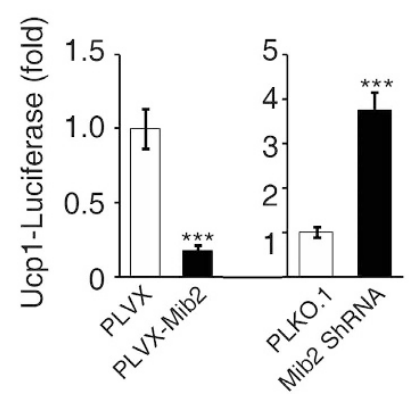

Cell Discovery | www.nature.com/celldisc 
ameliorated HFD-induced obesity, indicating that DJ-1 protein is detrimental for BAT activity. Furthermore, transplantation of DJ-1 Tg BAT failed to improve the functional integrity of endogenous BAT, confirming the function of DJ-1 in metabolic regulation. These results indicate that DJ-1 specifically modulates BAT activity to maintain energy and glucose homeostasis.

Ucp1, a gene selectively expressed in BAT, is localized in the inner mitochondrial membrane and is responsible for thermogenesis [26]. Ucp1 transcription is regulated by norepinephrine (NE), which is released by the sympathetic nervous system, and NE increases CREB/CEBP $\alpha$ complex-mediated Ucp1 expression in BAT [45]. In addition, other factors including Prdm16/ CEBP $\alpha$ [46], ER $\alpha$ [47], RIP40 [48] and FOXC2 [49] have also been reported to be involved in the regulation of Ucp1 expression. Recently, PTEN has been linked to the regulation BAT function [23, 25]. Here, we found that DJ-1 inhibits Ucp1 transcription and BAT activity in vitro and in vivo by negatively regulating PTEN signaling.

It has been reported that DJ-1 cooperates with Parkin and PINK1 to form the ubiquitin E3 ligase complex, promoting protein degradation [17]. Interestingly, Parkin and PINK1 both participate in the regulation of lipid metabolism $[18,19]$. Recently, it has been reported PTEN ubiquitination was mediated by E3 ligases, including Nedd4a [30], WWP2 [50], XIAP [51] and CHIP [52]. In our hands, none of the above reported E3 ligases was responsible for DJ-1-mediated PTEN protein degradation (data not shown). In the present work, we identified Mib2 as a new E3 ligase for PTEN degradation, which is involved in DJ-1mediated PTEN degradation in BAT and regulation of Ucp1 transcription. Mib2 is an E3 ligase in Notch [53] and NF- $\kappa B$ signaling [54]. Here we demonstrated that Mib2 regulates PI3K/Akt signaling through promoting PTEN degradation.

DJ-1 is reported to antagonize PTEN and mediate Akt activation in tumorigensis [15] and we found a conserved signaling cascade in the regulation of BAT function. We further found that Akt1 deletion increases Ucp1 expression in BAT. Interestingly, deletion of Akt1 in DJ-1 transgenic mice can rescue DJ-1 overexpression-induced Ucp1 inhibition and obesity. Hence, we have demonstrated that the DJ-1/Mib2/ PTEN/ PI3K/Akt/FoxO1 signaling pathway regulates Ucp1 transcription in BAT.

Insulin signaling promotes glucose tolerance through increasing Akt phosphorylation and increasing GLUT4 translocation [55]. In this study, improved glucose homeostasis, increased PTEN protein stability and decreased Akt phosphorylation were observed in DJ-1 KO mice. In contrast, we observed opposing phenotype in DJ-1 Tg mice. As a putative phosphatase, PTEN plays a dominant inhibitory role for PI3K/Akt signaling. Conditional deletion PTEN in mice has been shown to affect liver, muscle, adipose tissue and pancreatic beta cells to induced insulin hypersensitivity [56-59] and PTEN mutations cause constitutive insulin sensitivity in human [60]. These phenotypes mechanistically coincide with Akt2, but not Akt1 knockout mice. However, PTEN transgenic mice showed reduced body weight, elevated energy expenditure and increased insulin sensitivity [23]. In addition, Akt1 knockout mice have improved insulin sensitivity $[36,61]$, which is similar as that of PTEN transgenic mice. These findings indicate that isoforms of Akt or tissue specificity of PTEN proteins are critical for

Figure 5 DJ-1 promotes PTEN degradation through E3 ligase Mib2. (a) Lysates of BAT were immunoprecipitated with DJ-1 antibody followed by immunoblotting with Mib2 antibody. (b) Endogenous co-IP assay of Mib2 and PTEN was performed in wild-type or DJ-1 KO brown adipocytes. (c) The ubiquitination of Mib2 was examined in HEK293T cells after transfected with GFP-tagged Mib2, HA-tagged ubiquitin and Myc-tagged DJ-1 or their control vectors. After $16 \mathrm{~h}$ transfected, the cells were treated with MG132 for $8 \mathrm{~h}$. Cell lysates were immunoprecipitated with GFP antibody and immunoblotted with HA antibody. (d) The ubiquitination of PTEN was examined in HEK293T cells after transfected with the indicated plasmids. Cells were treated and analysis as in c. (e) Mib2 ubiquitination PTEN in vitro. His-PTEN was incubated with ubiquitin, E1, E2 (Ubc4) and ubiquitin ligase (Mib2, CHIP, Parkin), as indicated, and then it was analyzed by immunoblot using anti-His antibody. (f, g) Endogenous PTEN ubiqutination was examined in brown adipocytes with Mib2 overexpression (f) or Mib2 knockdown (g). Cells were treated with MG132 for $8 \mathrm{~h}$. Cell lysates were immunoprecipitated with PTEN antibody and immunoblotted with ubiquitin antibody. (h) HEK293T cells were transfected with Flag-tagged WT Mib2 or RING domain truncation ( $\triangle$ RING) Mib2 plasmids alone or together with HA-tagged ubiquitin, GFP-tagged PTEN. The cells then treated and analysis as in (c). (i) DJ-1 promotes Mib2 ubiquitination PTEN in vitro. His-PTEN was incubated with ubiquitin, E1, E2 (Ubc4), GST-DJ-1 and ubiquitin ligase (Mib2, CHIP and Parkin), as indicated, and then it was analyzed by immunoblot using anti-His antibody. GST-DJ-1 increased PTEN ubiqutination to 1.3-fold as indicated in the bottom of the panel. (j) Endogenous PTEN ubiqutination was examined in brown adipocytes with DJ-1-overexpression and/or Mib2 knockdown. Cells treated and analysis as in $\mathbf{f}$. (k) Lysates of brown adipocytes with Mib2 overexpression or knockdown were immunoblotted with the indicated antibodies. (I) Ucp1-luciferase activity was analyzed in brown adipocytes with Mib2 overexpression or knockdown. 

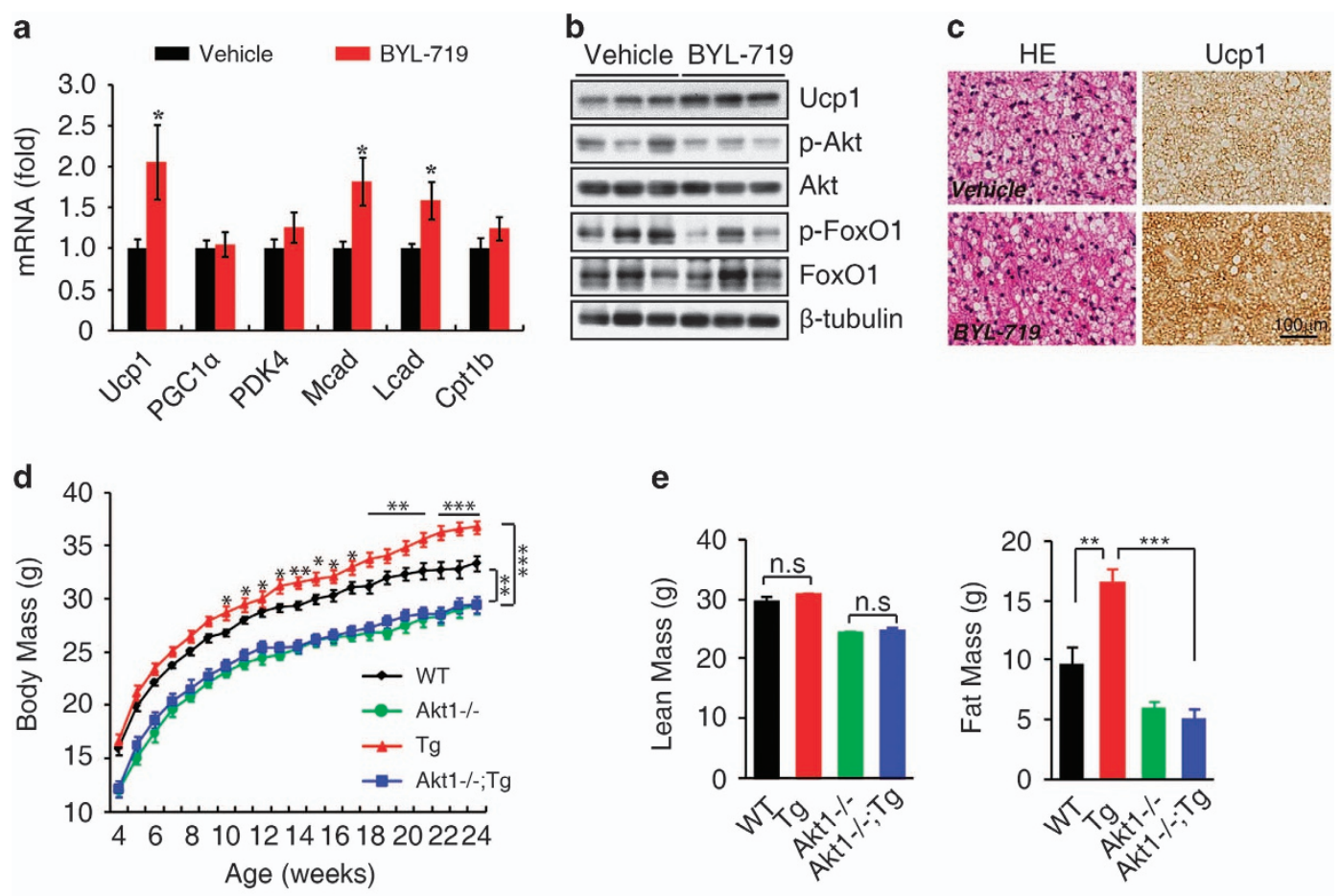

e
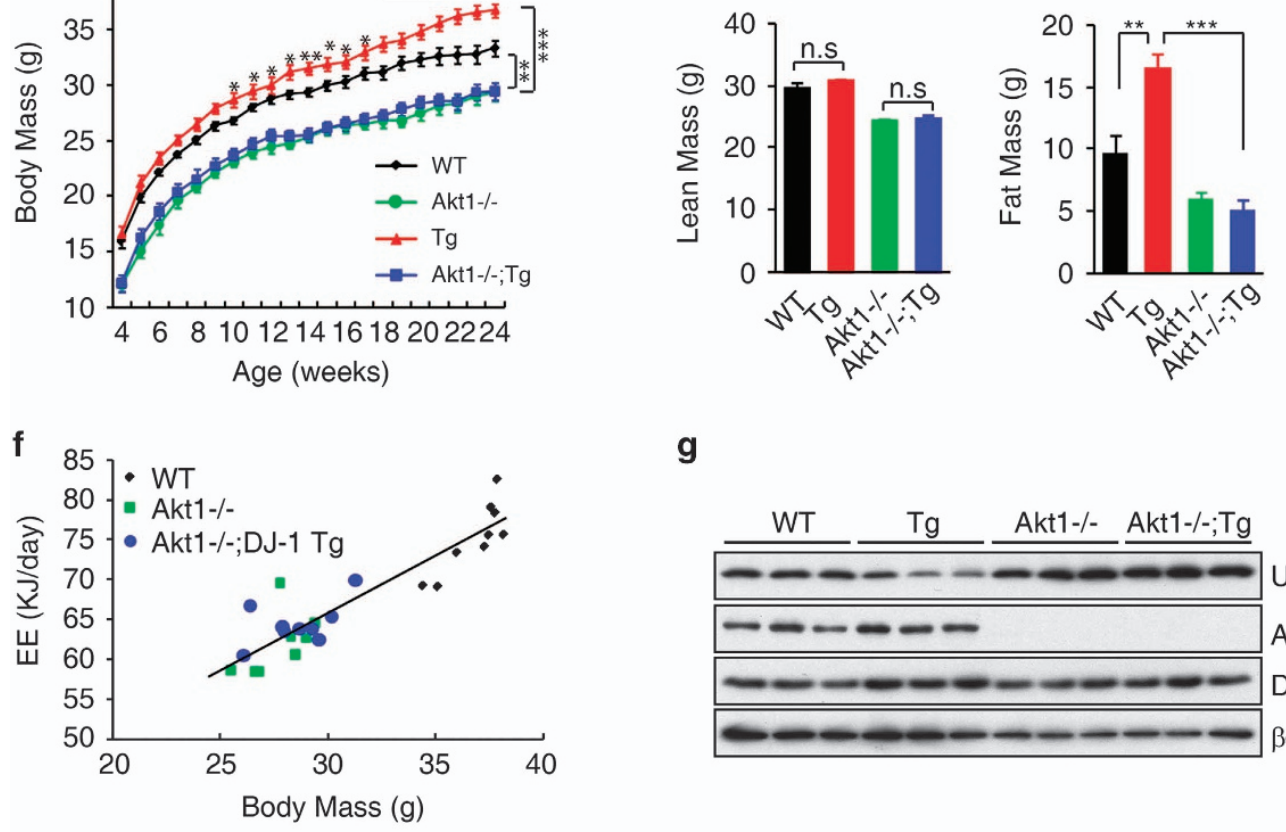

g

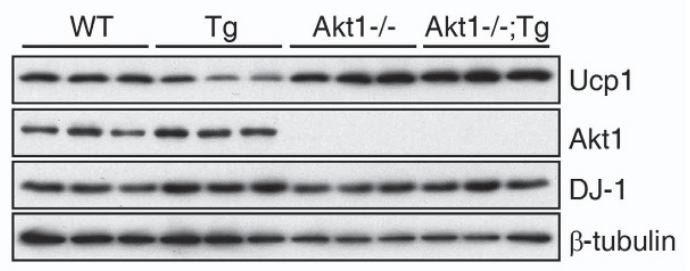

h
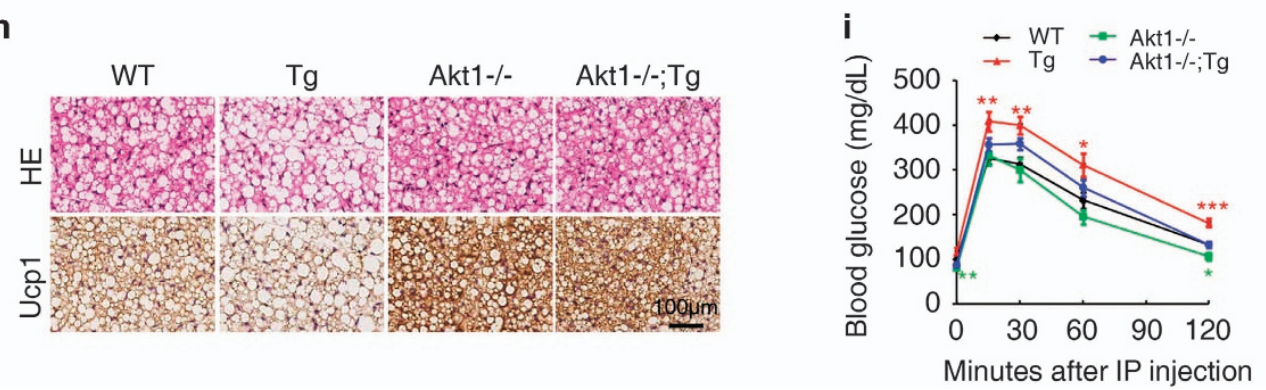

Figure 6 Ablation of Akt1 mitigates the obesity and BAT dysfunction induced by DJ-1 transgene. (a, b) The effect of BYL-719 on gene and protein expression in BAT. (c) HE or anti-Ucp1 antibody staining in BAT was performed after BYL-719 treatment. (d) Body mass of wild-type mice (WT), DJ-1 transgenic mice (Tg, Line 17 C57BL/6J background), Akt1 KO mice (Akt1 ${ }^{-/-}$), DJ-1 transgenic and Akt1 knockout mice (Akt1 ${ }^{-1-} ; \mathrm{Tg}$ ) fed on a chow diet for 24 weeks (WT, $n=15 ; \mathrm{Akt1}^{-/-}, n=9 ; \mathrm{Tg}, n=14 ; \mathrm{Akt1}{ }^{-/-}$; $\mathrm{Tg}, n=17)$. (e) Body lean and fat mass of 12-month-old indicated genotype mice measured by MRI (WT, $n=7 ; \mathrm{Akt} 1^{-1-}, n=11$; $\mathrm{Tg}, n=8$; Akt1 ${ }^{-1-} ; \mathrm{Tg}, n=8$ ). (f) Statistical analysis by General Linear Model (GLM) and ANCOVA analysis of the indicated genotype mice energy expenditure (EE) (WT, $n=9$; Akt1 ${ }^{-1-}, n=8$; Akt1 $1^{-1-} ; \mathrm{Tg}, n=9$ ). (g) Lysates of BAT from mice of the genotypes indicated immunoblotted with Ucp1, Akt1, DJ-1 or $\beta$-tubulin antibodies. (h) HE and Ucp1 staining of BAT from 4-weekold mice of the indicated genotypes. (i) GTT results for 9-month-old indicated genotype mice fed on a chow diet (WT, $n=8$; Tg, $n=7 ;$ Akt1 $^{-1-}, n=7$; Akt1 $\left.{ }^{-1-} ; \operatorname{Tg}, n=8\right)$. 
a

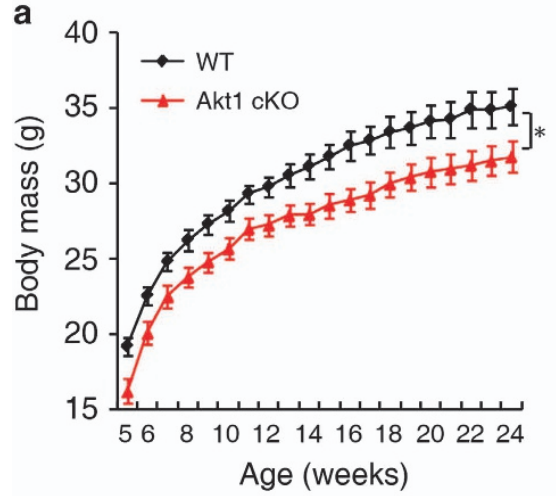

d

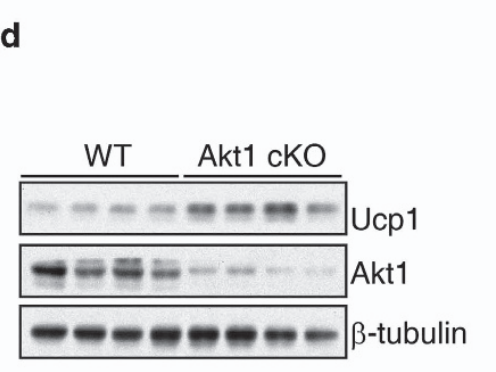

b



C

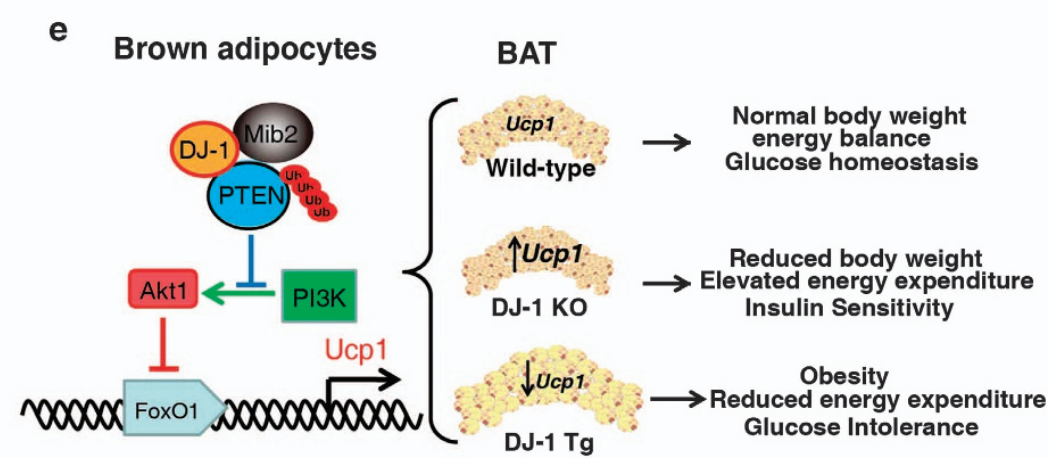

Figure 7 Specific knockout Akt1 in BAT increases Ucp1 expression. (a) Body mass of wild-type mice (WT) and Akt1 conditional knockout mice (Akt1 cKO) fed on a chow diet for 24 weeks(WT, $n=17$; Akt1 cKO, $n=16)$. (b) MRI analysis body lean and fat mass from 25-week-old Akt1 BAT-specific knockout (Akt1 cKO) and WT littermates mice (WT, $n=9$; Akt1 cKO, $n=9$ ). (c) HE and Ucp1 staining of sWAT and BAT from 4-week-old mice of the Akt1 cKO and WT mice. (d) Lysates of BAT from 4-week-old WT and Akt1 cKO mice were subjected to immunoblotting with indicated antibody. (e) Model depicting the role of the DJ-1-Mib2-PTENAkt1- FoxO1 signaling cascade in energy balance and glucose homeostasis of BAT.

the regulation of glucose metabolism. For example, we observed that deletion of Akt1, but not Akt2, significantly blocked DJ-1 transgene-induced glucose intolerance and obesity. Moreover, deletion of Akt1 with myf5-cre induced hyperactive BAT (Figure 7a-d). Interestingly, Lynes et al. reported specific ablation of the insulin receptor in the Myf5 lineage inhibited the differentiation of brown adipose progenitor cells. Myf5-cre-mediated insulin receptor deletion reduced BAT mass and UCP1 expression in BAT [62]. In addition, fat-specific knockout of the insulin receptor by the aP2-cre, which is mainly expressed in mature brown and white adipocytes [63], leads to the reduced white adipose tissue mass but no significant change in brown adipose tissue mass $[64,65]$. The inconsistent phenotypes of insulin receptor in different cre-mice indicate that insulin receptor signaling could affect BAT mass mainly through regulating BAT progenitor differentiation. Here we found that Myf5-cre-mediated Akt1 deletion promoted UCP1 expression in BAT. The different phenotypes between insulin receptor deletion and Akt1 knockout might be due to that Akt1 regulates UCP1 expression only in mature brown adipocytes, whereas insulin receptor affects the brown adipose progenitor cells. These findings indicate that DJ-1-mediated metabolic regulation is Akt1-dependent in mature BAT.

In summary, the present work contributes to our understanding of the complicated regulatory network of metabolism. Our exogenous BAT transplantation data indicate that manipulating DJ-1 levels in BAT could be a possible therapeutic avenue for the treatment of obesity and diabetes.

\section{Materials and Methods}

Mice

DJ-1 knockout mice with a C57BL/6/CBA background were a kind gift from Dr Jie Shen of Harvard Medical School. Mice used here were backcrossed with C57BL/6 mice at least five times. A Flag-tagged human DJ-1 open reading frame was used to generate DJ-1 transgenic mice in a CD-1 background. The line 17 was maintained in the C57BL/6 background by backcrossing at least eight times. Akt1 knockout 129/Sv/C57BL/6 background mice were backcrossed with C57BL/6J mice more 
than five times. Myf5 cre mice were a kind gift from Dr Dahai Zhu. Myf5 cre mice, Akt1 $1^{\text {flox/folx }}$ mice and Akt2 knockout mice were maintained in C57BL/6 J background. Littermate controls were used in all experiments, and animals were random allocated to experimental groups and processed.

Mice were housed at $22-24{ }^{\circ} \mathrm{C}$ in a $12 \mathrm{~h} / 12 \mathrm{~h}$ light-dark cycle with free access to water and were fed either with a standard chow diet or, when indicated, with a high-fat diet (45\% of total calories from fat).

Mice were maintained in the Animal Care Facility at the Institute of Biophysics, Chinese Academy of Sciences, Beijing, and all experiments involving animals were approved by the Institutional Animal Care and Use Committee.

\section{Energy intake, energy expenditure and activity measurement}

Mice were housed one animal per cage, and were given free access to food and water. Food intake and oxygen consumption were measured for two consecutive days using a TSE lab master system. Briefly, mice were placed in the measurement cages $4 \mathrm{~h}$ prior to data recording. Room temperature was maintained at $23{ }^{\circ} \mathrm{C}$ and light/dark cycles were of $12 \mathrm{~h}$. The volume of $\mathrm{O}_{2}$ consumed $\left(\mathrm{VO}_{2}\right)$ and $\mathrm{CO}_{2}$ eliminated $\left(\mathrm{VCO}_{2}\right)$ was recorded every $12 \mathrm{~min}$ (in six simultaneous metabolic chambers, with a sample period of $6 \mathrm{~min}$ per cage, plus $1 \mathrm{~min}$ purge per cage). The respiratory quotient (RQ) was calculated as: $\mathrm{RQ}=\mathrm{VCO}_{2} / \mathrm{VO}_{2}$. Energy expenditure (EE) was calculated as: $\mathrm{EE}=(3.815$ $\left.+(1.232 \times \mathrm{RQ})) \times \mathrm{VO}_{2} \times 1.44 \times 24 / 1000\right)$. Ambulatory activity of each mouse was measured using the optical beam technique (Opto-M3; Columbus Instruments, Columbus, OH, USA) over a period of two days and is expressed as 24-hour average activity.

\section{Brown adipose tissue transplantation}

After cervical dislocation of 5-week-old WT or DJ-1 KO donor mice, BAT was removed, peripheral white fat being excluded, and placed in sterile PBS. 0.2 $\mathrm{g}$ donor BAT was then transplanted as quickly as possible into the subcutaneous dorsal region, adjacent to the endogenous intra-scapular fat pad, of recipient mice. Recipient mice were anesthetized by intraperitoneal injection with avertin ( $400 \mathrm{mg} \mathrm{kg}^{-1}$ body weight). After transplantation, recipient mice and sham operation mice were given $200 \mu 11 \mathrm{U} \mathrm{ml}^{-1}$ penicillin by intramuscular injection for seven consecutive days. After 1 week, recipient mice were fed on a high-fat diet for 16 weeks. The BAT from WT or DJ-1 Tg mice was transplanted to DJ-1 Tg mice and followed with similar procedure as the above except chow diet.

\section{Glucolse tolerance tests and Insulin tolerance tests}

For glucose tolerance tests (GTT), animals were fasted for $16 \mathrm{~h}$ (1700-0900 hours) with free access to drinking water. The glucose level was assessed following intraperitoneal glucose injection $\left(2.0 \mathrm{~g} \mathrm{~kg}^{-1}\right)$. Serum glucose levels were determined immediately before and 15, 30, 60 and $120 \mathrm{~min}$ after glucose injection using a glucometer (OneTouch Ultra, Bayer, Berlin, Germany). For insulin tolerance tests (ITT), mice were fasted for $4 \mathrm{~h}$ (9:00-13:00) and then intraperitoneally injected with human insulin $\left(0.75 \mathrm{U} \mathrm{kg}^{-1}\right.$ for C57BL/6 $\mathrm{J}$ background mice and
$1.5 \mathrm{U} \mathrm{kg}^{-1}$ for CD-1 background mice). Blood glucose levels were determined immediately before and 15, 30, 60 and $120 \mathrm{~min}$ after insulin injection.

\section{Histological analysis}

Brown adipose tissue, white adipose tissue and livers were fixed overnight in 4\% paraformaldehyde, embedded in paraffin blocks and sectioned. Tissue sections were stained with hematoxilin/eosin or with anti-Ucp1 (ab10983, Abcam, Cambridge, UK) following standard procedures.

\section{PI3K inhibitors treatment}

For in vitro assays, $10 \mu \mathrm{M}$ LY294002, GDC0941, BYL-719 and $1 \mu \mathrm{M}$ PI103 were used to treat brown adipocytes precursor cells. All PI3K inhibitor were obtained from Selleck (Houston, TX, USA), and dissolved in DMSO. For in vivo experiments, BYL-719 dissolved in $N$-methyl pyrrolidone, polyethylene glycol 300, Kolliphor HS15, and water (10\%:30\%:20\%:40\%, v/v) and was orally administrated to two months old C57BL/6J male mice at a dose of $50 \mathrm{mg} \mathrm{kg}^{-1}$. Mice were anaesthetized and killed $8 \mathrm{~h}$ later and tissues were extracted and analyzed.

\section{Isolation, culture, immortalization and differentiation of brown adipocyte precursors}

Brown adipocyte precursors were isolated from newborn C57BL/6J mice by collagenase digestion and cultured in high glucose DMEM medium $(20 \%$ FBS, 20 mм HEPES, $1 \times$ penicillin/streptomycin). The cells were immortalized by infection with a retroviral vector encoding SV40 Large T antigen. Primary brown adipocyte precursors from newborn wild-type and DJ-1 transgenic mice were differentiated by growing in culture medium supplemented with $20 \mathrm{nM}$ insulin and $1 \mathrm{~nm}$ T3 (differentiation medium). After reaching confluence, cells were cultured for $48 \mathrm{~h}$ in differentiation medium further supplemented with $0.5 \mathrm{~mm}$ isobutylmethylxanthine, $\quad 0.5 \mathrm{~mm}$ dexamethasone and $0.125 \mathrm{~mm}$ indomethacin. After culturing for 4 more days in differentiation medium, cells exhibited a fully differentiated phenotype with massive accumulation of multilocular fat droplets.

\section{Quantitative RT-PCR}

Total RNA from tissues or cells was extracted using TRIZOL (Invitrogen, Waltham, MA, USA). Reverse transcription was performed using random primers. Quantitative real time-PCR was performed using TransStart Green qPCR SuperMix (Transgen Biotecth, Beijing, China) in a Stratagene Mx3005P (Agilent Technologies, Santa Clara, CA ,USA). Primer sequences are given below. The housekeeping gene used for input normalization of all the qPCR data was $\beta$-actin. mRNA expression was measured by quantitative PCR using the DeltaDelta CT method. Primers for quantitative PCR: $\beta$-actin-Fw: 5'-GGCTGTATTCCCCTCCATCG-3'; $\beta$-actin-Rv: 5'-CCAG TTGGTAACAATGCCATGT-3'; Ucp1-Fw: 5'-AGG CTTCCAGTACCATTGGT-3'; Ucp1-Rv: 5'-CTGAGTGA GGCAAAGCTGATT T-3'; PGC1 $\alpha-\mathrm{Fw}$ : 5'-TATGGAGTGA CATAGAGTGTGCT-3'; PGC1 $\alpha$-Rv: 5'-CCACTTCAATCC ACCCAGAAAG-3'; PDK4-Fw: 5'- 
AGGGAGGTCGAGCTGTTCTC-3'; PDK4-Rv: 5'GGAGTGTTCACTAAGCGGTCA-3'; Prdm16-Fw: 5'CCACCAGCGAGGACTTCAC-3'; Prdm16-Rv: 5'-GGA GGACTCTCGTAGCTCGAA-3'; CPT1b-Fw: 5'-GCACA CCAGGCAGTAGCTTT-3'; CPT1b-Rv: 5'-CAGGAGTTG ATTCCAGACAGGTA-3'; Mcad-Fw: 5'-AGGGTTTAGTT TTGAGTTGACGG-3'; Mcad-Rv: 5'-CCCCGCTTTTGT CATATTCCG-3'; Lcad-Fw: 5'-TCTTTTCCTCGGAG CATGACA-3'; Lcad-Rv: 5'-GACCTCTCTACTCACTTCT CCAG-3'; Cebpo-Fw: 5'-CAAGAACAGCAACGAGTACCG -3'; Cebpo-Rv: 5'-GTCACTGGTCAACTCCAGCAC-3'; Cebp $\beta$-Fw: 5'-CAAGCTGAGCGACGAGTACA-3'; Cebp $\beta$ Rv: 5'-AGCTGCTCCACCTTCTTCTG-3'; DJ-1-Fw: 5'-GCT TCCAAAA GAGCTCTGGTC-3'; DJ-1-Rv: 5'-ACATCACG GCTACACTG-3'; PTEN-Fw: 5'-TGGATTCGACTTAGAC TTGACCT-3'; PTEN-Rv: 5'-GCGGTGTCATAATGTCTC TCAG-3'; TH-Fw: 5'-CCAAGGTTCATTGGACGGC-3'; TH-Rv: 5'-CTCTCCTCGAATACCACAGCC-3'; Agrp-Fw: 5'-ATGCTGACTGCAATGTTGCTG-3'; Agrp-Rv: 5'-CAGA CTTAGACCTGGGAACTCT-3'; Npy-Fw: 5'-ATGCTAGG TAACAAGCGAATGG-3'; Npy-Rv: 5'-TGTCGCAGAGC GGAGTAGTAT-3'; Pomc-Fw: 5'-ATGCCGAGATTCTGCT ACAGT-3'; Pomc-Rv: 5'-CCACACATCTATGGAGGTCT GAA-3'; Stat3-Fw: 5'-CAATACCATTGACCTGCCGAT-3'; Stat3-Rv: 5'-GAGCGACTCAAACTGCCCT-3'; Leprb-Fw: 5'-TGGTCCCAGCAGCTATGGT-3'; Leprb-Rv: 5'-ACCCA GAGAAGTTAGCACTGT-3'.

\section{Plasmids construct and screen stable expression cell}

DNA fragments corresponding to full-length or $\triangle \mathrm{RING}$ (deletion of the RING domain) Mib2 (isoform2) were amplified from a mouse brown adipose precursors cDNA library by PCR and inserted into $\mathrm{p} 3 \times$ FLAG-CMV-10 Expression Vector (Sigma-Aldrich, St Louis, MO, USA) using the NotI and EcoRI restriction sites. FLAG-HA tagged DJ-1(mouse) was inserted retroviral vector PQCXIH using the NotI and BamHI restriction sites.

generate DJ-1 overexpression cells, immortalized brown fat precursors were infected with the retroviral vector PQCXIH encoding FLAG-HA-DJ-1 (mouse) and selected with hygromycin. To generate DJ-1 stable knockdown cells, immortalized brown fat precursors were infected the lentiviral vector PLKO.1 encoding short hairpin RNA for mouse DJ-1. shRNA targeting sequences used were shRNA1\#: 5'-ATCTGGGTGCACAGA ATTTAT-3'; and shRNA2\#: 5'-CCATACGATGTGGTGG TTCTT-3'. Mib2 stable knockdown cell used shRNA targeting sequences TCGAAGGATGAAGAAGTGTAT by the similar methods. To generate Mib2 stable overexpression cell, immortalized brown fat precursors were infected with the lentiviral vector PLVX-AcGFP-N1 encoding FLAG-Mib2 (mouse) and selected with puromycin.

\section{Co-immunoprecipitation}

Cells for co-immunoprecipitation were lysed in IP buffer containing $50 \mathrm{~mm}$ Hepes, $\mathrm{pH} 7.9,150 \mathrm{~mm} \mathrm{NaCl}, 10 \%$ Glycerol, $1 \%$ Triton-100, $1.5 \mathrm{~mm} \mathrm{MgCl}_{2}, 0.1 \mathrm{~m} \mathrm{NaF}, 1 \mathrm{~mm}$ EGTA, $2 \mathrm{~mm}$ phenylmethylsulfonyl fluoride, $2 \mu \mathrm{g} \mathrm{ml}^{-1}$ aprotinin and leupeptin and $1 \mathrm{~mm}$ sodium vanadate. Lysates were centrifuged at
$12000 \mathrm{~g}$ for $15 \mathrm{~min}$ at $4{ }^{\circ} \mathrm{C}$ prior to immunoprecipitation and precleared with $2 \mu \mathrm{IgG}$ and protein $\mathrm{G}$ agarose beads at $4{ }^{\circ} \mathrm{C}$ for $2 \mathrm{~h}$. Following the removal of the beads by centrifugation, lysates were incubated with appropriate antibodies in the presence of $30 \mu \mathrm{l}$ of protein $\mathrm{G}$ agarose beads for at least $2 \mathrm{~h} 4^{\circ} \mathrm{C}$. The beads were washed with IP buffer four times and the immunoprecipitates were subjected to immunoblotting.

\section{Immunoblotting}

Tissues or cells were for immunoblotting were lysed in buffer containing $50 \mathrm{~mm}$ Hepes, pH 7.4, $150 \mathrm{~mm} \mathrm{NaCl}, 1 \%$ Nonidet P-40, 0.1\% deoxycholate, $0.05 \%$ SDS, $0.1 \mathrm{~m} \mathrm{NaF}, 1$ mм EGTA, $2 \mathrm{~mm}$ phenylmethylsulfonyl fluoride, $2 \mu \mathrm{g} \mathrm{ml}^{-1}$ aprotinin and leupeptin and $1 \mathrm{~mm}$ sodium vanadate. Protein concentration was determined using a Bio-Rad protein assay kit (Bio-Rad, Hercules, California, USA). Proteins were separated on a $10 \%$ polyacrylamide gel and transferred to a methanol-activated PVDF membrane (GE Healthcare, Little Chalfont, UK). The membrane was blocked for one hour in TBST containing 5\% milk and subsequently probed with primary antibodies overnight at $4{ }^{\circ} \mathrm{C}$. After incubating for $1 \mathrm{~h}$ with goat-anti-mouse or goat-antirabbit HRP-conjugated secondary antibodies (GE Healthcare), protein level was detected with Super Signal West Pico and Femto Luminol reagents (Thermo Scientific, Waltham, MA, USA). The antibodies used were anti-phosphor-Akt (Ser473; \#4060, Cell Signaling, Cambridge, MA, USA), anti-Akt (\#4691, Cell Signaling), anti-Akt1 (\#2938, Cell Signaling), anti-FoxO1 (sc-11350, Santa Cruz, Dallas, TX, USA), anti-Akt2 (\#3063, Cell Signaling), anti-phosphor-FoxO (Thr24-FoxO1/ Thr32-FoxO3a; \#9464, Cell Signaling), anti-phosphor-HSL (Ser660; \#4126, Cell Signaling), anti-HSL (\#4107, Cell Signaling), anti-PTEN (\#9188, Cell Signaling), anti-DJ-1 (\#5933, Cell Signaling), anti-Ucp1 (ab10983), anti-Ubiquitin (\#3936, Cell Signaling), anti-Mib2 (Ls-C295379, LifeSpan BioSciences, Seattle, WA, USA), antiFLAG (Sigma), anti-GFP (Invitrogen), anti-HA (Santa Cruz), anti- $\beta$-tubulin (CW0098A, CWBiotech, Beijing, China), anti- $\beta$ actin (60008-1-Ig, Proteintech Group, Campbell Park, Chicago, IL, USA) and anti-GAPDH (CW0266A, CWBiotech).

\section{In vivo PTEN ubiquitylation assays}

For PTEN ubiquitination assays, the cells were treated with $20 \mu \mathrm{g} \mathrm{ml}^{-1}$ of the proteasome inhibitor MG132 (Selleck) for $8 \mathrm{~h}$. Then, the cells were lysed in buffer containing $50 \mathrm{~mm}$ Hepes, $\mathrm{pH}$ 7.4, $150 \mathrm{~mm} \mathrm{NaCl}, 1 \%$ Nonidet P-40, $0.1 \%$ deoxycholate, $0.05 \%$ SDS, $0.1 \mathrm{M} \mathrm{NaF}, 1 \mathrm{~mm}$ EGTA, $2 \mathrm{~mm}$ phenylmethylsulfonyl fluoride, $2 \mu \mathrm{g} \mathrm{ml}^{-1}$ aprotinin and leupeptin, $1 \mathrm{~mm}$ sodium vanadate and $20 \mu \mathrm{g} \mathrm{ml}^{-1} \mathrm{MG132}$. The lysates were centrifuged and supernatant were incubate with PTEN or GFP antibody for $4 \mathrm{~h}$ and protein $\mathrm{G}$ beads for further $2 \mathrm{~h}$. Then the beads were washed 4 times with lysis buffer, following by SDS-PAGE electrophoresis and immunoblotting.

\section{In vitro ubiquitination}

The reaction was carried out at $37^{\circ} \mathrm{C}$ for $1 \mathrm{~h}$ in $50 \mu \mathrm{l}$ reaction buffer (20 mм Tris- $\mathrm{HCl}$, pH 7.4, $1 \mathrm{~mm}$ DTT, $10 \mathrm{~mm} \mathrm{MgCl}_{2}$, $1 \mathrm{~mm}$ ATP) containing the following components: $0.5 \mu \mathrm{g}$ of ubiquitin, $250 \mathrm{ng} \mathrm{E1}, 500 \mathrm{ng}$ of E2( Ubc4) and $0.5 \mu \mathrm{g}$ of His-PTEN. FLAG-tagged E3 ligase were purification from 
HEK293T cells which transfected with FLAG-tagged Mib2, CHIP or Parkin. After ubiquitination reaction, the samples were directly boiled with SDS-PAGE loading buffer and analyzed by immunoblotting against anti-HA antibody (Santa Cruz). The samples were separated on a $8 \%$ SDS-PAGE followed by immunoblot analysis using His antibody (CW0286, CWBiotech).

\section{Luciferase reporter assay}

The Ucp1-luciferase reporter gene was a kind gift from Dr Mark Christian of the University of Warwick. $0.5 \mu \mathrm{g}$ Ucp1-luciferase reporter genes and $0.2 \mu \mathrm{g}$ TK-Renilla were co-transfected into pre-brown adipocytes cells in a 24 -well plate using lipofectamin-2000 (Invitrogen). After $24 \mathrm{~h}$, cell extracts were obtained and firefly and Renilla luciferase activities were measured using a Promega Dual-Luciferase reporter system.

\section{Chromatin immunoprecipitation}

Mice were perfused with normal salting following $37 \%$ formaldehyde after starvation $24 \mathrm{~h}$ at $8{ }^{\circ} \mathrm{C}$. BAT was removed, peripheral white fat being excluded, and placed in $1 \mathrm{ml}$ cell lysis buffer ( 5 mM Pipes $(\mathrm{KOH}), \mathrm{pH} 8.0,85 \mathrm{~mm} \mathrm{KCl}$, Nonidet P-40 $(0.5 \% \mathrm{vol} / \mathrm{vol}), 1 \times$ protease inhibitors], tissue were cut into small pieces by scissors following homogenates by homogenizer. Removed supernatant and collected nuclear pellet by centrifugation and lysed in $300 \mu \mathrm{l}$ nuclear lysis buffer $(1 \%$ (w/v) SDS, 5 mм EDTA, $50 \mathrm{~mm}$ Tris. $\mathrm{Cl}$, $\mathrm{pH} 8.0,1 \times$ protease inhibitors). The mixture was then sonicated to produce soluble chromatin with an average size of 300-1500 bp. After centrifugation at $12000 \times \mathrm{g}$ for $20 \mathrm{~min}$, the supernatant was collected. Soluble chromatin was diluted 10-fold in dilution buffer [1.11\% Triton X-100, $1.67 \mathrm{~mm}$ EDTA, $167 \mathrm{~mm} \mathrm{NaCl}$, $16.7 \mathrm{~mm}$ Tris. $\mathrm{Cl}, \mathrm{pH} 8.0,1 \times$ protease inhibitor] and then precleared by incubating $1 \mathrm{ml}$ diluted chromatin with salmon protein-A agarose beads ( $50 \mu 150 \%$ slurry in $10 \mathrm{~mm}$ Tris. $\mathrm{Cl}, \mathrm{pH}$ $8.1,1 \mathrm{~mm}$ EDTA) for $2 \mathrm{~h}$ at $4{ }^{\circ} \mathrm{C}$. The supernatant was collected by centrifugation, $20 \%$ of which was retained for use as the total input control. $4 \mu \mathrm{g}$ ChIP-grade anti-FoxO1 (ab39670, Abcam) antibody and $2 \mu \mathrm{g}$ anti-FoxO1 (sc-11350, Santa Cruz) antibody was added to the lystaes and incubated overnight at $4{ }^{\circ} \mathrm{C}$ with agitation. To collect the immunocomplex, $60 \mu \mathrm{l}$ of salmon protein-A agarose beads were added to the samples and incubated for $4 \mathrm{~h}$ at $4{ }^{\circ} \mathrm{C}$. The beads were collected and washed sequentially for $5 \mathrm{~min}$ each in low salt wash buffer $[0.1 \% \mathrm{SDS}$, 1\% Triton X-100, 2 mм EDTA, $20 \mathrm{~mm}$ Tris $\mathrm{pH} 8.0,150 \mathrm{~mm}$ $\mathrm{NaCl}, 1 \times]$, high salt wash buffer $[0.1 \%$ SDS, $1 \%$ Triton $\mathrm{X}-100$, 2 mм EDTA, 20 mм Tris, pH 8.0, 500 mм NaCl, $3 \times$ ), $\mathrm{LiCl}$ buffer $(0.25 \mathrm{~m} \mathrm{LiCl}, 1 \%$ Nonidet P-40, $1 \%$ deoxycholate, $10 \mathrm{~mm}$ Tris. $\mathrm{Cl}, 1 \mathrm{~mm}$ EDTA; $2 \times)$ and Tris-EDTA buffer $(10 \mathrm{~mm}$ Tris $\mathrm{pH} 8.0,1 \mathrm{~mm}$ EDTA, $2 \times)$. The bound protein-DNA immunocomplexes were eluted with $250 \mu$ l elution buffer ( $1 \%$ SDS, $0.1 \mathrm{M}$ $\mathrm{NaHCO}_{3}$ ) and reversed formaldehyde cross-links by adding $1 \mu \mathrm{l}$ $10 \mathrm{mg} \mathrm{ml}^{-1} \mathrm{RNase}$ and $5 \mathrm{M} \mathrm{NaCl}$ to a final concentration of $0.3 \mathrm{M}$ to eluants and incubating in a $65^{\circ} \mathrm{C}$ water bath for $5 \mathrm{~h}$. After adding 2.5 volumes of $100 \%$ ethanol and precipitating overnight at $-20^{\circ} \mathrm{C}$, DNA was pelleted and resuspended in $100 \mu \mathrm{l}$ of water. $2 \mu \mathrm{l}$ of $0.5 \mathrm{~m}$ EDTA, $4 \mu \mathrm{l} 1 \mathrm{~m}$ Tris, $\mathrm{pH} 6.5$ and $1 \mu \mathrm{l}$ of $20 \mathrm{mg} \mathrm{ml}^{-1}$ Proteinase $\mathrm{K}$ were added and incubated for $2 \mathrm{~h}$ at $55^{\circ} \mathrm{C}$. DNA was purified using a QIAquick PCR Purification Kit (Qiagen) and eluted in $50 \mu \mathrm{l}$ TE buffer. Two microliter of the eluted DNA sample was used in $\mathrm{qPCR}$ reactions. The primers used for amplifications were as follows:

Ucp1 promoter (forward): 5'- CTGTTGTTGCTGCTGCT GTT-3'

Ucp1 promoter (reverse): 5'- GGAAGCTGCAAGACCT ATGG-3'

\section{Statistical analysis}

Comparisons between two groups were made by unpaired two-tailed Student's $t$-tests. Data for body mass change over time were analyzed by an unbalanced two-way analysis of variance (ANOVA). All values are expressed as mean \pm s.e.m. $* P<0.05,{ }^{*} P<0.01, * * * P<0.001$ for two-tailed Student's $t$-tests, unless stated otherwise.

\section{Conflict of Interest}

The authors declare no conflict of interest.

\section{Acknowledgements}

We thank the members of the Yuan laboratory for critical reading of the manuscript and helpful discussion. We thank the Pathology Core Facility in the Institute of Biophysics, CAS. We also thank Dr Joyce Flemmings for the English editing. This work was supported by the grants from the strategic priority research program (XDB13030000 to WJ), the National Science Foundation of China (Grant No. 81125010 and 81030025 to ZY), the National Basic Research Program of China (973-2012CB910701 and 2013DFA31990 to ZY) and Crossdisciplinary Collaborative Teams Program for Science, Technology and Innovation (2014-2016) from Chinese Academy of Sciences; and Key research program (KJZD-EW-L01-3 to WJ), One Hundred Talents Program (WJ) of the Chinese Academy of Sciences and from the Ministry of Science and Technology of China (2012CBA01301 and 2012CB944701 to WJ), as well as by a grant from the National Natural Science Foundation of China (31171131 and 81370951 to WJ).

\section{Author contributions}

RW, GN, WJ and ZY designed the research; RW, XML, JGS, HC, JM, MD, SP and JQW performed the research; RW, XML, JQD, JRS, GN, DHL, WJ and ZY analyzed the data; and RW, XML, WJ and ZY wrote the paper.

\section{References}

1 Tseng YH, Cypess AM, Kahn CR. Cellular bioenergetics as a target for obesity therapy. Nat Rev Drug Discov 2010; 9: 465-482.

2 Jin W, Patti ME. Genetic determinants and molecular pathways in the pathogenesis of Type 2 diabetes. Clin Sci (Lond) 2009; 116: 99-111. 
3 van Marken Lichtenbelt WD, Vanhommerig JW, Smulders NM et al. Cold-activated brown adipose tissue in healthy men. N Engl J Med 2009; 360: 1500-1508.

4 Cypess AM, Lehman S, Williams G et al. Identification and importance of brown adipose tissue in adult humans. $N$ Engl J Med 2009; 360: 1509-1517.

5 Gunawardana SC, Piston DW. Reversal of type 1 diabetes in mice by brown adipose tissue transplant. Diabetes 2012; 61: $674-682$.

6 Stanford KI, Middelbeek RJ, Townsend KL et al. Brown adipose tissue regulates glucose homeostasis and insulin sensitivity. J Clin Invest 2013; 123: 215-223.

7 Liu X, Zheng Z, Zhu X et al. Brown adipose tissue transplantation improves whole-body energy metabolism. Cell Res 2013; 23: 851-854.

8 Wagenfeld A, Yeung CH, Shivaji S, Sundareswaran VR, Ariga H, Cooper TG. Expression and cellular localization of contraception-associated protein. J Androl 2000; 21: 954-963.

9 Okada M, Matsumoto K, Niki T, Taira T, Iguchi-Ariga SM, Ariga H. DJ-1, a target protein for an endocrine disrupter, participates in the fertilization in mice. Biol Pharm Bull 2002; 25: 853-856.

10 Yoshida K, Sato Y, Yoshiike M, Nozawa S, Ariga H, Iwamoto T. Immunocytochemical localization of DJ-1 in human male reproductive tissue. Mol Reprod Dev 2003; 66: 391-397.

11 Takahashi K, Taira T, Niki T, Seino C, Iguchi-Ariga SM, Ariga $\mathrm{H}$. DJ-1 positively regulates the androgen receptor by impairing the binding of PIASx alpha to the receptor. $J$ Biol Chem 2001; 276: 37556-37563.

12 Pitkanen-Arsiola T, Tillman JE, Gu G et al. Androgen and anti-androgen treatment modulates androgen receptor activity and DJ-1 stability. Prostate 2006; 66: 1177-1193.

13 Niki T, Takahashi-Niki K, Taira T, Iguchi-Ariga SM, Ariga H. DJBP: a novel DJ-1-binding protein, negatively regulates the androgen receptor by recruiting histone deacetylase complex, and DJ-1 antagonizes this inhibition by abrogation of this complex. Mol Cancer Res 2003; 1: 247-261.

14 Nagakubo D, Taira T, Kitaura H et al. DJ-1, a novel oncogene which transforms mouse NIH3T3 cells in cooperation with ras. Biochem Biophys Res Commun 1997; 231: 509-513.

15 Kim RH, Peters M, Jang Y et al. DJ-1, a novel regulator of the tumor suppressor PTEN. Cancer Cell 2005; 7: 263-273.

16 Bonifati V, Rizzu P, van Baren MJ et al. Mutations in the DJ-1 gene associated with autosomal recessive early-onset parkinsonism. Science 2003; 299: 256-259.

17 Xiong H, Wang D, Chen L et al. Parkin, PINK1, and DJ-1 form a ubiquitin E3 ligase complex promoting unfolded protein degradation. J Clin Invest 2009; 119: 650-660.

18 Kim KY, Stevens MV, Akter MH et al. Parkin is a lipidresponsive regulator of fat uptake in mice and mutant human cells. J Clin Invest 2011; 121: 3701-3712.

19 Franks PW, Scheele C, Loos RJ et al. Genomic variants at the PINK1 locus are associated with transcript abundance and plasma nonesterified fatty acid concentrations in European whites. FASEB J 2008; 22: 3135-3145.
20 Yang Y, Gehrke S, Haque ME et al. Inactivation of Drosophila DJ-1 leads to impairments of oxidative stress response and phosphatidylinositol 3-kinase/Akt signaling. Proc Natl Acad Sci USA 2005; 102: 13670-13675.

21 Vasseur S, Afzal S, Tardivel-Lacombe J, Park DS, Iovanna JL, Mak TW. DJ-1/PARK7 is an important mediator of hypoxia-induced cellular responses. Proc Natl Acad Sci USA 2009; 106: 1111-1116.

22 Di Cristofano A, Pesce B, Cordon-Cardo C, Pandolfi PP. Pten is essential for embryonic development and tumour suppression. Nat Genet 1998; 19: 348-355.

23 Ortega-Molina A, Efeyan A, Lopez-Guadamillas E et al. Pten positively regulates brown adipose function, energy expenditure, and longevity. Cell Metab 2012; 15: 382-394.

24 Garcia-Cao I, Song MS, Hobbs RM et al. Systemic elevation of PTEN induces a tumor-suppressive metabolic state. Cell 2012; 149: 49-62.

25 Sanchez-Gurmaches J, Hung CM, Sparks CA, Tang Y, Li H, Guertin DA. PTEN loss in the Myf5 lineage redistributes body fat and reveals subsets of white adipocytes that arise from Myf5 precursors. Cell Metab 2012; 16: 348-362.

26 Cannon B, Nedergaard J. Brown adipose tissue: function and physiological significance. Physiol Rev 2004; 84: 277-359.

27 Harms M, Seale P. Brown and beige fat: development, function and therapeutic potential. Nat Med 2013; 19: 1252-1263.

28 Liu X, Wang S, You Y et al. Brown adipose tissue transplantation reverses obesity in $\mathrm{Ob} / \mathrm{Ob}$ mice. Endocrinology 2015; 156: 2461-2469.

29 Wang X, Trotman LC, Koppie T et al. NEDD4-1 is a protooncogenic ubiquitin ligase for PTEN. Cell 2007; 128: 129-139.

30 Trotman LC, Wang X, Alimonti A et al. Ubiquitination regulates PTEN nuclear import and tumor suppression. Cell 2007; 128: 141-156.

31 Fritsch C, Huang A, Chatenay-Rivauday C et al. Characterization of the novel and specific PI3Kalpha inhibitor NVPBYL719 and development of the patient stratification strategy for clinical trials. Mol Cancer Ther 2014; 13: 1117-1129.

32 Easton RM, Cho H, Roovers K et al. Role for Akt3/protein kinase Bgamma in attainment of normal brain size. Mol Cell Biol 2005; 25: 1869-1878.

33 Cho $\mathrm{H}, \mathrm{Mu}$ J, Kim JK et al. Insulin resistance and a diabetes mellitus-like syndrome in mice lacking the protein kinase Akt2 (PKB beta). Science 2001; 292: 1728-1731.

34 Garofalo RS, Orena SJ, Rafidi K et al. Severe diabetes, agedependent loss of adipose tissue, and mild growth deficiency in mice lacking Akt2/PKB beta. J Clin Invest 2003; 112: 197-208.

35 Chen WS, Xu PZ, Gottlob K et al. Growth retardation and increased apoptosis in mice with homozygous disruption of the Akt1 gene. Genes Dev 2001; 15: 2203-2208.

36 Wan M, Easton RM, Gleason CE et al. Loss of Akt1 in mice increases energy expenditure and protects against dietinduced obesity. Mol Cell Biol 2012; 32: 96-106.

37 Schulz TJ, Huang P, Huang TL et al. Brown-fat paucity due to impaired BMP signalling induces compensatory browning of white fat. Nature 2013; 495: 379-383. 
38 Jain D, Jain R, Eberhard D et al. Age- and diet-dependent requirement of DJ-1 for glucose homeostasis in mice with implications for human type 2 diabetes. $\mathrm{J} \mathrm{Mol} \mathrm{Cell} \mathrm{Biol}$ 2012; 4: 221-230.

39 Kim JM, Jang HJ, Choi SY et al. DJ-1 contributes to adipogenesis and obesity-induced inflammation. Sci Rep 2014; 4: 4805.

40 Yamaguchi S, Yamane T, Takahashi-Niki K et al. Transcriptional activation of low-density lipoprotein receptor gene by DJ-1 and effect of DJ-1 on cholesterol homeostasis. PLoS One 2012; 7: e38144.

41 Shi SY, Lu SY, Sivasubramaniyam T et al. DJ-1 links muscle ROS production with metabolic reprogramming and systemic energy homeostasis in mice. Nat Commun 2015; 6: 7415.

42 Yoneshiro T, Aita S, Matsushita M et al. Recruited brown adipose tissue as an antiobesity agent in humans. The Journal of clinical investigation 2013; 123: 3404-3408.

43 Surwit RS, Wang S, Petro AE et al. Diet-induced changes in uncoupling proteins in obesity-prone and obesityresistant strains of mice. Proc Natl Acad Sci USA 1998; 95: 4061-4065.

44 Echtay KS, Roussel D, St-Pierre J et al. Superoxide activates mitochondrial uncoupling proteins. Nature 2002; 415: 96-99.

45 Ortega-Molina A, Serrano M. PTEN in cancer, metabolism, and aging. Trends in endocrinology and metabolism: TEM 2013; 24: 184-189.

46 Kajimura S, Seale P, Kubota K et al. Initiation of myoblast to brown fat switch by a PRDM16-C/EBP-beta transcriptional complex. Nature 2009; 460: 1154-1158.

47 Park CJ, Zhao Z, Glidewell-Kenney C et al. Genetic rescue of nonclassical ERalpha signaling normalizes energy balance in obese Eralpha-null mutant mice. The Journal of clinical investigation 2011; 121: 604-612.

48 Debevec D, Christian M, Morganstein D et al. Receptor interacting protein 140 regulates expression of uncoupling protein 1 in adipocytes through specific peroxisome proliferator activated receptor isoforms and estrogen-related receptor alpha. Mol Endocrinol 2007; 21: 1581-1592.

49 Cederberg A, Gronning LM, Ahren B, Tasken K, Carlsson $\mathrm{P}$, Enerback S. FOXC2 is a winged helix gene that counteracts obesity, hypertriglyceridemia, and diet-induced insulin resistance. Cell 2001; 106: 563-573.

50 Maddika S, Kavela S, Rani N et al. WWP2 is an E3 ubiquitin ligase for PTEN. Nat Cell Biol 2011; 13: 728-733.

51 Van Themsche C, Leblanc V, Parent S, Asselin E. X-linked inhibitor of apoptosis protein (XIAP) regulates PTEN ubiquitination, content, and compartmentalization. J Biol Chem 2009; 284: 20462-20466.

52 Ahmed SF, Deb S, Paul I et al. The chaperone-assisted E3 ligase $\mathrm{C}$ terminus of Hsc70-interacting protein (CHIP) targets PTEN for proteasomal degradation. J Biol Chem 2012; 287: 15996-16006.

53 Koo BK, Yoon KJ, Yoo KW et al. Mind bomb-2 is an E3 ligase for Notch ligand. J Biol Chem 2005; 280: 22335-22342.

54 Stempin CC, Chi L, Giraldo-Vela JP, High AA, Hacker H, Redecke V. The E3 ubiquitin ligase mind bomb-2 (MIB2) protein controls B-cell CLL/lymphoma 10 (BCL10)- dependent NF-kappaB activation. J Biol Chem 2011; 286: 37147-37157.

55 Bryant NJ, Govers R, James DE. Regulated transport of the glucose transporter GLUT4. Nat Rev Mol Cell Biol 2002; 3: 267-277.

56 Stiles B, Wang Y, Stahl A et al. Liver-specific deletion of negative regulator Pten results in fatty liver and insulin hypersensitivity [corrected]. Proc Natl Acad Sci USA 2004; 101: 2082-2087.

57 Wijesekara N, Konrad D, Eweida M et al. Muscle-specific Pten deletion protects against insulin resistance and diabetes. Mol Cell Biol 2005; 25: 1135-1145.

58 Kurlawalla-Martinez C, Stiles B, Wang Y, Devaskar SU, $\mathrm{Kahn} \mathrm{BB}, \mathrm{Wu} \mathrm{H}$. Insulin hypersensitivity and resistance to streptozotocin-induced diabetes in mice lacking PTEN in adipose tissue. Mol Cell Biol 2005; 25: 2498-2510.

59 Stiles BL, Kuralwalla-Martinez C, Guo W et al. Selective deletion of Pten in pancreatic beta cells leads to increased islet mass and resistance to STZ-induced diabetes. Mol Cell Biol 2006; 26: 2772-2781.

60 Pal A, Barber TM, Van de Bunt M et al. PTEN mutations as a cause of constitutive insulin sensitivity and obesity. $N$ Engl J Med 2012; 367: 1002-1011.

61 Buzzi F, Xu L, Zuellig RA et al. Differential effects of protein kinase $\mathrm{B} / \mathrm{Akt}$ isoforms on glucose homeostasis and islet mass. Mol Cell Biol 2010; 30: 601-612.

62 Lynes MD, Schulz TJ, Pan AJ, Tseng YH. Disruption of insulin signaling in Myf5-expressing progenitors leads to marked paucity of brown fat but normal muscle development. Endocrinology 2015; 156: 1637-1647.

63 Barlow C, Schroeder M, Lekstrom-Himes J et al. Targeted expression of Cre recombinase to adipose tissue of transgenic mice directs adipose-specific excision of loxP-flanked gene segments. Nucleic Acids Res 1997; 25: 2543-2545.

64 Bluher M, Michael MD, Peroni OD et al. Adipose tissue selective insulin receptor knockout protects against obesity and obesity-related glucose intolerance. Dev Cell 2002; 3: 25-38.

65 Katic M, Kennedy AR, Leykin I et al. Mitochondrial gene expression and increased oxidative metabolism: role in increased lifespan of fat-specific insulin receptor knockout mice. Aging Cell 2007; 6: 827-839.

(Supplementary Information is linked to the online version of the paper on the Cell Discovery website.)

(i) This work is licensed under a Creative Commons Attribution 4.0 International License. The images or other third party material in this article are included in the article's Creative Commons license, unless indicated otherwise in the credit line; if the material is not included under the Creative Commons license, users will need to obtain permission from the license holder to reproduce the material. To view a copy of this license, visit http://creativecommons.org/licenses/by/4.0/

(C) The Author(s) 2017 